Article

\title{
Structural Damage Detection with Different Objective Functions in Noisy Conditions Using an Evolutionary Algorithm
}

\author{
Faisal Shabbir ${ }^{1, *}$ (D), Muhammad Imran Khan ${ }^{2,3}$, Naveed Ahmad ${ }^{1}$, Muhammad Fiaz Tahir ${ }^{1}$ (D), \\ Naeem Ejaz ${ }^{1}$ and Jawad Hussain ${ }^{1}$ \\ 1 Department of Civil Engineering, University of Engineering and Technology, Taxila 47050, Pakistan; \\ n.ahmad@uettaxila.edu.pk (N.A.); fiaz.tahir@uettaxila.edu.pk (M.F.T.); naeem.ejaz@uettaxila.edu.pk (N.E.); \\ jhus015@aucklanduni.ac.nz (J.H.) \\ 2 Department of Electronics Engineering, University of Engineering and Technology, Taxila 47050, Pakistan; \\ imran@alumni.chalmers.se \\ 3 Micro/Nano Electronic System Integration R\&D Center (MESIC), University of Science \& Technology of \\ China (USTC), Hefei 230027, China \\ * Correspondence: fsha049@aucklanduni.ac.nz; Tel.: +92-331-816-4351
}

Received: 12 October 2017; Accepted: 28 November 2017; Published: 1 December 2017

\begin{abstract}
Dynamic properties such as natural frequencies and mode shapes are directly affected by damage in structures. In this paper, changes in natural frequencies and mode shapes were used as the input to various objective functions for damage detection. Objective functions related to natural frequencies, mode shapes, modal flexibility and modal strain energy have been used, and their performances have been analyzed in varying noise conditions. Three beams were analyzed: two of which were simulated beams with single and multiple damage scenarios and one was an experimental beam. In order to do this, SAP 2000 (v14, Computers and Structures Inc., Berkeley, CA, United States, 2009) is linked with MATLAB (r2015, The MathWorks, Inc., Natick, MA, United States, 2015). The genetic algorithm (GA), an evolutionary algorithm (EA), was used to update the damaged structure for damage detection. Due to the degradation of the performance of objective functions in varying noisy conditions, a modified objective function based on the concept of regularization has been proposed, which can be effectively used in combination with EA. All three beams were used to validate the proposed procedure. It has been found that the modified objective function gives better results even in noisy and actual experimental conditions.
\end{abstract}

Keywords: dynamic properties; objective functions; evolutionary algorithm; regularization; noisy conditions

\section{Introduction}

Structural identification of constructed systems typically requires the integration of structural conceptualization, finite element (FE) modeling, experimental execution, data processing, model calibration, simulation, interpretation and decisions [1]. Experimental responses are related to the physical properties of the system, and changes in these physical quantities are reflected as a change in the experimental responses. Therefore, in order to identify the actual physical characteristics of structures, it is necessary to carry out field tests and measure the resulting responses to correlate and validate the associated FE models. Both static and dynamic tests can be carried out on the actual structure for structure assessment.

FE models are mathematical models that provide a means of predicting characteristic responses of structures without actually building them. In FE models, the continuous domain of the actual physical 
structure with infinite degrees of freedom (DOF) is discretized into small components called FEs. These models have been extensively used in industrial and research applications as they give a reasonable representation of the actual structure, but they are prone to certain inaccuracies and errors [2]. Structure health monitoring (SHM) is defined as tracking the structural responses, possibly along with inputs, over a sufficiently long duration to determine deterioration, anomalies and damage in a structure, in order to make optimal decisions for asset management [3]. More specifically, the responses of the system should be measured in such a way that any operational incidents, damage or anomalies that can affect the serviceability and reliability of the structure are detected [4]. Model updating is a branch of optimization wherein the differences in the experimental responses and their FE model counterparts are minimized using mathematical techniques.

Generally, in model updating and damage assessment problems, not only is a good correlation required between experimental and analytical results, but also the updated parameters should have physical significance. The success of model updating is dependent on three key factors, setting up of the objective function, the selection of parameters and a robust optimization algorithm [5]. A proper objective function is necessary to effectively minimize the difference between the experimental and analytical results. Selection of parameters is an important issue as there may be many sets of parameters that can produce acceptable results. A robust optimization algorithm is required to find the minimum in the search space. The algorithm should be able to find a global minimum out of many local minima with precision and accuracy.

The key step is to define residual vectors for the experimental and analytical values in terms of experimental frequencies and mode shapes. The residual vectors lead to objective functions that can contain different criteria for finding out the discrepancies between the experiment and FE model such as differences of frequencies, model coordinates, modal assurance criteria (MAC), coordinate modal assurance criteria (COMAC) and others [6]. It is the goal of optimization to minimize the error residual to determine a set of physically-justifiable parameters [7]. The outcome of a model updating application to a constructed system should be an analytical model that can replicate the actual mechanical characteristics of the built structure with a good level of confidence.

Damage detection of civil structures is a difficult optimization problem. The algorithm capability and search domain complexity are the two main aspects to be considered [8]. The sensitivity method (SM) is traditionally used for model updating and damage assessment, but it is prone to be attracted to local minima, which may lead to a solution with no physical significance. In certain cases, manual model updating has to be done in SM, and even then, the global solution is not confirmed [9]. Different objective functions have also been tried using SM. Commonly-used objective functions using SM include both natural frequencies and MAC-based objective functions [10]. Further, FE model updating and damage detection have also been tried with SM using objective functions based on strain energy residuals [11] and modal flexibility [12].

To alleviate the issues associated with SM being attracted to local minima instead of global minima in difficult optimization problems, global optimization algorithms (GOAs) have received the attention of researchers [13]. The main difference between the two approaches is that SM works on a single point, whereas GOAs work in parallel on many points consecutively [14,15]. Evolutionary algorithms (EAs) are part of GOAs, which deal with mechanisms inspired by biological evolution such as cross over, mutation and selection. In the context of model updating and damage detection, several studies have been conducted using different objective functions with GOAs such as genetic algorithm (GA) [15-18], simulated annealing (SA) [19] and particle swarm optimization (PSO) [20]. GA was applied to a simulated beam, as well as a frame structure using frequencies and MAC-based objective functions to efficiently detect damage [16,17]. Multiobjective GA was also used to detect damage in a simulated beam and signature bridge structure based on MAC and flexibility-based objective functions [21]. However, due to the simplification of a 3D model to a 2D model, the first three vertical modes were matched, and torsional modes were not included in model updating. Furthermore, multiobjective multicriteria GA was studied for damage detection and estimation using MAC and 
flexibility-related objective functions [18]. A numerical beam and wing plate experimental structure was also investigated using both GA and SA with the frequency response function (FRF)-based objective function [19], and a new bended SA algorithm was also proposed for improvement of the results. Damage detection in thin plates based on modal data using a PSO-GA-based approach has been presented by Vaez and Fallah [22]. In a study, breeder GA was also used for an adaptive FE model for dome structures [23]. Another study [24] proposed hybrid GA and SA to improve the solution and convergence. A multiobjective formulation has also been developed for FE model updating using GA [25]. PSO was also used for assessment of beam and truss structures using the frequency-based modal residual $[20,26]$. Modeling errors were also investigated using GA and PSO in a multiobjective sense for damage estimation using frequency and mode shape residuals [27].

Ill-conditioning is a topic of concern in the FE model updating procedure. In the case of an ill-conditioned system and measurement noise, the system parameters in different sets can produce the same set of responses at distinct locations of the search space; hence, the solution cannot be affirmed as unique. Three remedies are introduced to overcome the ill-conditioning of the problem, i.e.: (i) modification of the space of the system parameters; (ii) modification of measured data; and (iii) modification of the error function [28]. The first two remedies are about the relationship between the number of parameters and measured data. The first remedy calls for a reduction in the number of uncertain parameters for model updating. The most commonly-used approach is grouping similar elements into one parameter [29]. Efforts to increase the number of independent vibration responses have been made in pursuit of the second remedy [30] by using laser vibrometry or by testing the structure in different configurations [31].

Diverse inverse problems have been attempted by adopting the third remedy, called a regularization technique [28,32]. Regularization techniques make use of the prior information to get an additional check for the minimization problem by modifying the error function. The unknown parameters are assumed to be closer to the known nominal values. The solution space, for example, is directly reduced by setting the upper limits in close proximity to the baseline characteristics. It is done by incorporating a weighted norm of the parameter changes to keep the parameter changes small. The most usual form is known as the Tikhonov regularization [33], as it was introduced to resolve the ill-conditioning of systems with the aim to control the condition number of the problem. Addressing the ill-conditioning using a regularization technique is a topic of interest in FE model updating problems. Sensitivity-based regularization techniques have been explored in this context by various researchers. Ahmadian et al. [34] addressed the problem by incorporating side constraints and used singular value decomposition, generalized cross-validation (GCV) and L curves for the determination of the regularization parameter. The $\mathrm{L}$ curve approach and the generalized singular value approach are used reliably to get updated parameters with physical understanding. Weber et al. [35] investigated structural damage detection with Tikhonov regularization, and Weber et al. [36] introduced consistent regularization for a non-linear model updating problem. Further studies of regularization techniques in model updating include Titurus and Friswell [37] and Mottershead et al. [38]. In these studies, the sensitivity-based updating with a special focus on optimization of the response prediction and a priori information about the uncertain parameters has been investigated with the help of an additional regularization criterion. Chen and Muang [39] investigated dynamic perturbation theory for incomplete noisy modal data and used the L-curve approach for regularization parameter determination. Entezami et al. [40] proposed a new sensitivity-based damage detection method in which an improved sensitivity function related to modal strain energy and modified GCV is presented. Grip et al. [41] proposed a new regularization method that is based on the minimization of total variation on sensitivity-based model updating.

GA, an EA, has been used in this research to exploit the search space fully instead of sensitivity-based local search optimization. For efficient damage detection, an effective objective function is needed to match the experimental data with the analytical predictions. Therefore, to harness the benefits associated with EA and to handle ill-conditioning using the concept of regularization, 
this paper aims to propose a new objective function using EA. Initially, the performance of different objective functions has been compared for damage detection in noisy conditions to select the best objective function. The updated parameters may not turnout to be necessarily realistic in noisy conditions, thus making damage detection difficult. Therefore, an additional regularization criterion has been proposed especially in noisy conditions to assess the damage correctly. In Section 2, theories related to objective functions, noise additions, mass normalization and regularization using EA are detailed. In Section 3, the different objective functions are compared in terms of their efficiency in damage assessment of two simulated beams and one experimental beam, and the proposed regularization has been applied. The results are analyzed and conclusions are drawn from the current study finally.

\section{Theoretical Background}

To assess the quality of the analytical model with respect to experimental data, correlation is normally the first step. It is a usual practice to correlate the analytical model results with experimental results to see if the analytical model is in reasonable agreement and can be updated in a reasonable way [42]. In dynamic modal updating, the modal data of the FE model are compared with modal data obtained from the experiment. The following are the commonly-used techniques to correlate the analytical and experimental data (such as frequencies and mode shapes) for model updating.

\subsection{Objective Functions}

In this research, a combination of objective functions related to the natural frequencies and mode shapes has been used.

\subsubsection{Natural Frequency}

The frequency difference between the experimental and analytical frequencies is given as:

$$
F_{1}=\sum_{i=1}^{n}\left[\left(\omega_{a, i}-\omega_{e, i}\right) / \omega_{e, i}\right]^{2}
$$

where $n$ is total number of modes, subscript $a$ and $e$ represent analytical and experimental counterparts, respectively, $n$ is the number of measured modes and $\omega$ is the natural frequency of the mode in question.

\subsubsection{Mode Shape}

Numerous studies have indicated that the location of damage can be detected using the changes in mode shape. MAC is used to compare the modal vectors quantitatively. Its value is limited between zero and one, with one representing fully-consistent mode shapes and zero representing inconsistent mode shapes.

For two modes $\boldsymbol{\phi}_{j}$ and $\boldsymbol{\phi}_{j}^{*}$, the MAC value is given as:

$$
\mathrm{MAC}=\frac{\left|\boldsymbol{\phi}_{j}^{\prime} \boldsymbol{\phi}_{j}^{*}\right|^{2}}{\left(\boldsymbol{\phi}_{j}^{\prime} \boldsymbol{\phi}_{j}\right)\left(\boldsymbol{\phi}_{j}^{* \prime} \boldsymbol{\phi}_{j}^{*}\right)}
$$

where $\boldsymbol{\phi}_{j}$ and $\boldsymbol{\phi}_{j}^{*}$ are the mode shapes of an undamaged and a damaged structure, respectively. For a given structure, if a MAC value obtained from a comparison of two mode shapes deviates from one, it can be taken as an indication of damage in the structure.

The MAC-related objective function used in the current study is defined [43] as:

$$
F_{2}=\sum_{i=1}^{n} \frac{\left(1-\sqrt{\mathrm{MAC}_{i}}\right)^{2}}{\mathrm{MAC}_{i}}
$$


The correlation between two modes and the use of MAC values have been outlined by Allemang [2]. For a shell structure, Srinivasan and Kot [3] found that if mode shape changes, it gives a more sensitive indication of damage as compared to a change in resonant frequencies.

\subsubsection{Modal Flexibility}

The dynamically-measured flexibility matrix can be used to estimate the damage in the actual structure. It actually is the inverse of the stiffness matrix and replicates the applied force and structural displacement relationship. Each column represents nodal displacements when a unit force is applied at one of the DOF. The flexibility matrix may be calculated from the lower vibration modes as:

$$
\begin{gathered}
\boldsymbol{F}=\frac{1}{\omega_{j}^{2}} \boldsymbol{\phi}_{j} \boldsymbol{\phi}_{j}^{\prime} \\
\boldsymbol{F}^{*}=\frac{1}{\omega_{j}^{* 2}} \boldsymbol{\phi}_{j}^{*} \boldsymbol{\phi}_{j}^{* \prime}
\end{gathered}
$$

where $\boldsymbol{F}$ and $\boldsymbol{F}^{*}$ are the flexibility matrices of a undamaged and a damaged structure, $\omega_{j}$ is the frequency of a particular mode, $\phi$ is the mass normalized mode shape and asterisks show properties of the damaged structure. The diagonal values of the matrices obtained using Equations (4) and (5) has been taken, and flexibility based modal assurance criteria (FMAC) is formulated as:

$$
\mathrm{FMAC}=\frac{\left|\boldsymbol{\varphi}_{j} \boldsymbol{\varphi}_{j}^{*}\right|^{2}}{\left(\boldsymbol{\varphi}_{j}^{\prime} \boldsymbol{\varphi}_{j}\right)\left(\boldsymbol{\varphi}_{j}^{* \prime} \boldsymbol{\varphi}_{j}^{*}\right)}
$$

where $\varphi_{j}$ and $\varphi_{j}^{*}$ represent the diagonal values of the flexibility matrices obtained from Equations (4) and (5), respectively.

The objective function is then formulated to calculate FMAC between $\boldsymbol{F}$ and $\boldsymbol{F}^{*}$ as:

$$
F_{3}=\sum_{i=1}^{n} \frac{\left(1-\sqrt{\mathrm{FMAC}_{i}}\right)^{2}}{\mathrm{FMAC}_{i}}
$$

where FMAC represents the change in the flexibility matrix due to damage formulated in light of Equation (3).

\subsubsection{Strain Energy}

Another index sensitive to damages is modal strain energy (MSE). In this method, damage in a region is located between two structural DOFs if a decrease in strain energy is caused. A change in strain energy has an inverse relation with the damage in the structure. MSE for a particular mode for a healthy and a damaged structure is given as:

$$
\begin{aligned}
\mathrm{MSE} & =\boldsymbol{\phi}_{j}^{\prime} \boldsymbol{K} \boldsymbol{\phi}_{j} \\
\mathrm{MSE}^{*} & =\boldsymbol{\phi}_{j}^{* \prime} \boldsymbol{K} \boldsymbol{\phi}_{j}^{*}
\end{aligned}
$$

where MSE is the healthy-simulated strain energy for the $j$-th mode shape and MSE* is thedamage-simulated strain energy for the $j$-th mode shape.

Therefore, for the undamaged and damaged structure, the difference in MSE is shown as:

$$
F_{4}=\sum_{i=1}^{n}\left|\boldsymbol{\phi}_{i}^{* \prime} \boldsymbol{K} \boldsymbol{\phi}_{i}^{*}-\boldsymbol{\phi}_{i}^{\prime} \boldsymbol{K} \boldsymbol{\phi}_{i}\right|
$$


The combined objective function is given as:

$$
\begin{aligned}
& \prod_{1}=F_{1}+F_{2} \\
& \prod_{\text {II }}=F_{1}+F_{3} \\
& \prod_{\text {III }}=F_{1}+F_{4}
\end{aligned}
$$

Minimization of these functions will maximize the correlation of the experimental and analytical model. Furthermore, the Objective Functions II and III require mode shapes to be expanded (to address the mismatch between the DOF in the FE model and experimental data), which has been done using the expansion using modal data (EMD) technique [42].

\subsubsection{Noise Addition and Mass Normalization}

To investigate the behavior of different objective functions in noisy conditions, random noise has been added as:

$$
\Phi_{j}=\Phi_{j}\left(1+\alpha_{\text {noise }} \varepsilon\right)
$$

where $\alpha_{\text {noise }}$ is the degree of noise being added, $\varepsilon$ represents a random number between -1 and +1 , and ' $j$ ' is the component to which noise has been added. The approach has been investigated in eight noise conditions, i.e., $1 \%, 3 \%, 5 \%$ and $10 \%$ in both frequencies and mode shapes and $1 \%, 3 \%, 5 \%$ and $10 \%$ in mode shapes only [16].

The random noise was added using the MATLAB command rand, which uses a standard uniform distribution. Furthermore, for noise addition of $1 \%$ to the modal data, the $\alpha_{\text {noise }}$ value has been taken as 0.01 in Equation (12). The noise has been added in the frequencies and mode shapes with respect to the noise-free case. Furthermore, the investigations were done in the frequency domain instead of the time domain in this paper; therefore, noise has been directly added to the frequency domain data.

Normalization is a process in which scale factors have been applied to mode shapes, such that they can be standardized (scale factors are sometimes applied to natural modes to standardize their elements associated with various DOFs). In certain cases, top floor displacement is taken as unity, whereas in other cases, the largest modal amplitude is taken as unity. However, in this research, mass-normalized mode shapes have been obtained as:

$$
M_{n}=\phi^{\prime} M \phi
$$

such that $\phi^{\prime} \boldsymbol{M} \phi=I$ where $I$ is the identity matrix. The resulting modes are orthogonal, as well as normalized with respect to mass matrix $\boldsymbol{M}$.

\subsubsection{Damage Parameterization}

In this work, single beam elements have been considered for structural representation, and uncertain physical properties were considered as updating parameters. No mass reduction has been assumed as is acceptable in most real-life damage detection studies. The damage index $d$ can be represented in terms of the bending stiffness of an individual element. Therefore, relative variation of the bending stiffness of the damaged element $E I_{d}$ compared to the initial estimated value $E I_{r}$ (reference model) according to damage mechanics [44] is given as:

$$
d=1-\frac{E I_{d}}{E I_{r}}
$$

This definition is advantageous as not only can it estimate the damage severity, but also its location at the element level. Although the damage index usually takes values between zero and one, this formulation can be used between the values of -1 and 1 , thus acting more like a correction factor to the stiffness of the elements. Therefore, the formulation can be effectively used for model updating of a reference model, as well as for the damage severity estimation. 


\subsubsection{Regularization in Model Updating Using Evolutionary Algorithm (EA)}

Regularization techniques in the context of model updating have been conventionally applied by various researchers using SM as explained in the literature review. However, in the context of this paper, where EA has been used to better explore the search domain, a regularization technique was combined with EA. Regularization usually attempts to control the parameter variations. In this research, the parameter values with respect to the original model are kept small during subsequent iterations. In other words, the physical assumption of the original model has been considered [37] such that the parameter values remain close to the original FE model. Therefore, the minimization term can be modified to give preference to a solution as:

$$
\prod_{\text {damaged.reg }}=\prod_{\text {damaged }}+\tau\left\|x-x_{o}\right\|
$$

where $x$ refers to the parameter values in the current iteration and $x_{0}$ refers to the initial parameter values. $\tau$ is the tradeoff parameter between the two parts of the objective function. For a small $\tau$, the updated parameters are almost unrestricted, and the solution resembles the original ill-posed problem. However, for a large value, the updating parameters remain limited in size and may have larger errors in the fitting of the data.

\section{Damage Detection Case Studies}

In order to investigate different objective functions for the model updating problem, three beams were considered. Two beams were simulated beams, and one was an experimental beam. The first simulated beam is only damaged at one location, and the second simulated beam is damaged at more than one location to check the effectiveness of objective functions at various noise levels. For the third beam, experimental data are available for damage [45]. The effectiveness of the three objective functions is then evaluated on both simulated and experimental data. Only the first three mode shapes and model frequencies are considered, as it is likely that only a few modes can be identified during actual experiments. The FE model updating procedure and damage assessment are carried out in the MATLAB environment.

\subsection{Simulated Simply Supported Beam 1 (Case 1)}

The simulated beam has a rectangular cross-section of $0.25 \times 0.20 \mathrm{~m}$ and a 6-m length (Figure 1a). It was equally divided into 10 two-dimensional beam elements. The values for other material properties corresponding to reinforced cement concrete were taken as $2500 \mathrm{~kg} / \mathrm{m}^{3}$ for density and $32 \mathrm{GPa}$ for elastic modulus. The constitutive laws assumed for the beam were linear perfectly elastic. Case 1 with the single damage scenario is considered (Figure 1b). A number of approaches can be used to model cracks in beams. In this research, the cracks were simulated by reducing the stiffness of a particular element of the structure locally [46]. This approach can approximate the stiffness changes for beams with open cracks under low frequency vibrations, as compared to breathing cracks, which open and close and produce non-linear dynamics [47]. In the beam, only the sixth element of the beam was considered to be damaged. A $20 \%$ reduction in the moment of inertia (MOI) has been assumed as compared to the reference model for damage detection at different noise levels. This leads to a value of 0.8 for the ratio $E I_{d} / E I_{r}$ in Equation (12) and a corresponding damage index of 0.2. Only the first three lower natural frequencies and mode shapes have been used in the current research. The mode shapes were expanded and then mass normalized using Equation (13). No modeling or mesh errors were assumed in the simulated study (Case 1 and Case 2) as the simulations were aimed at observing the performance of different objective functions on damage assessment.

The general background theory of GA can be found elsewhere [48]. GA implemented in MATLAB was used in this research. GA was used to minimize the Objective Functions I, II and III with noise. Eight noise cases have been considered: noise both in frequencies and mode shapes at $1 \%, 3 \%, 5 \%$ and $10 \%$ and noise only in mode shapes at $1 \%, 3 \%, 5 \%$ and $10 \%$. A population of 100 , maximum iterations 
of 500, tournament selection with tournament size of 4 , two-point cross over with $70 \%$ probability, mutation with a probability of $1 \%$ and a threshold for minimum change in five consecutive generations was set as $10^{-10}$.The upper bound for all the parameters was set at 1.15-times the initial model, and the lower bound was set as 0.25 -times the initial model parameter values as it can be assumed that the frequencies decreased due to damage in the elements.

INITIAL MODEL

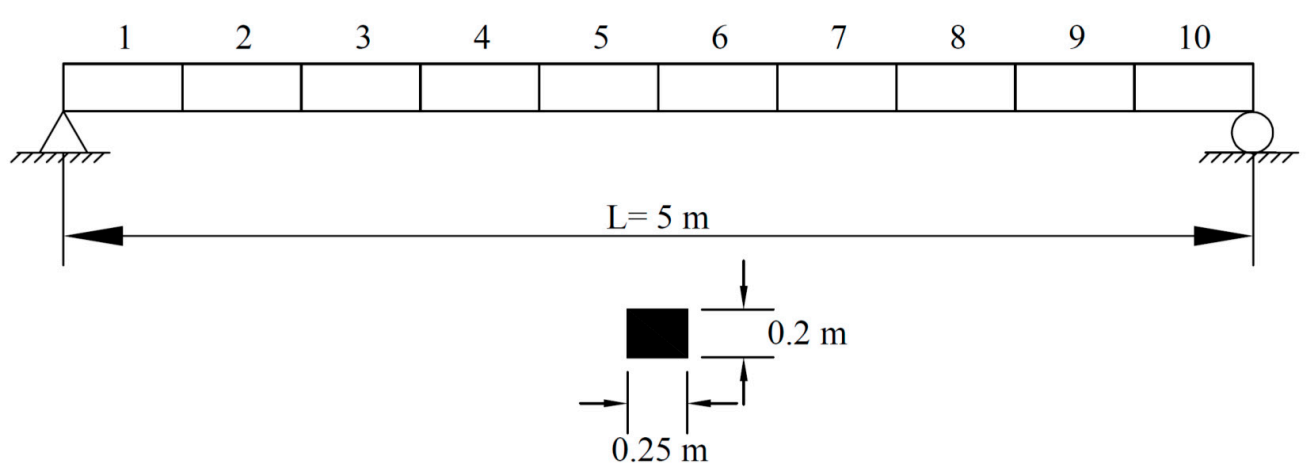

DAMAGED MODEL (Case-1)

(a)

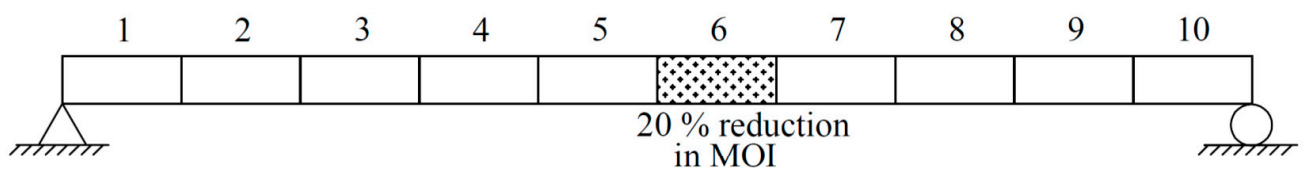

DAMAGED MODEL (Case-2)

(b)



(c)

Figure 1. (a) Simulated healthy beam. (b) Damaged model (Case 1) withsingle damage scenario. (c) Damaged model (Case 2) with multiple damage scenario. MOI, moment of inertia.

The damage distribution for Case 1 is reported in Figure 2 using Objective Function I. Furthermore, to check the effect of noise on frequencies and mode shapes, two noise cases were investigated, wherein noise was added both in frequency and mode shapes (Figure 2a) and noise in mode shapes only (Figure 2b). Likewise, Figures 3 and 4 represent the damage distribution for Case 1 using Objective Functions II and III.

It has been noted that all the objective functions detected the damage correctly with $0 \%$ noise in the measurement. However, it can also be noticed that when the noise level increases, the damage detection capabilities of all the objective functions are degraded. Only Objective Function I performed better than the other two combinations. Wrong detection of damage in other elements is more pronounced in Objective Functions II and III at higher noise levels (above 1\%). The results are nearly pointless in these cases, as the damages in other elements exceeded the original damage level in Element No. 6. Therefore, it can be concluded that Objective Function I (Figure 2) performed better than the other two (Figures 3 and 4). 


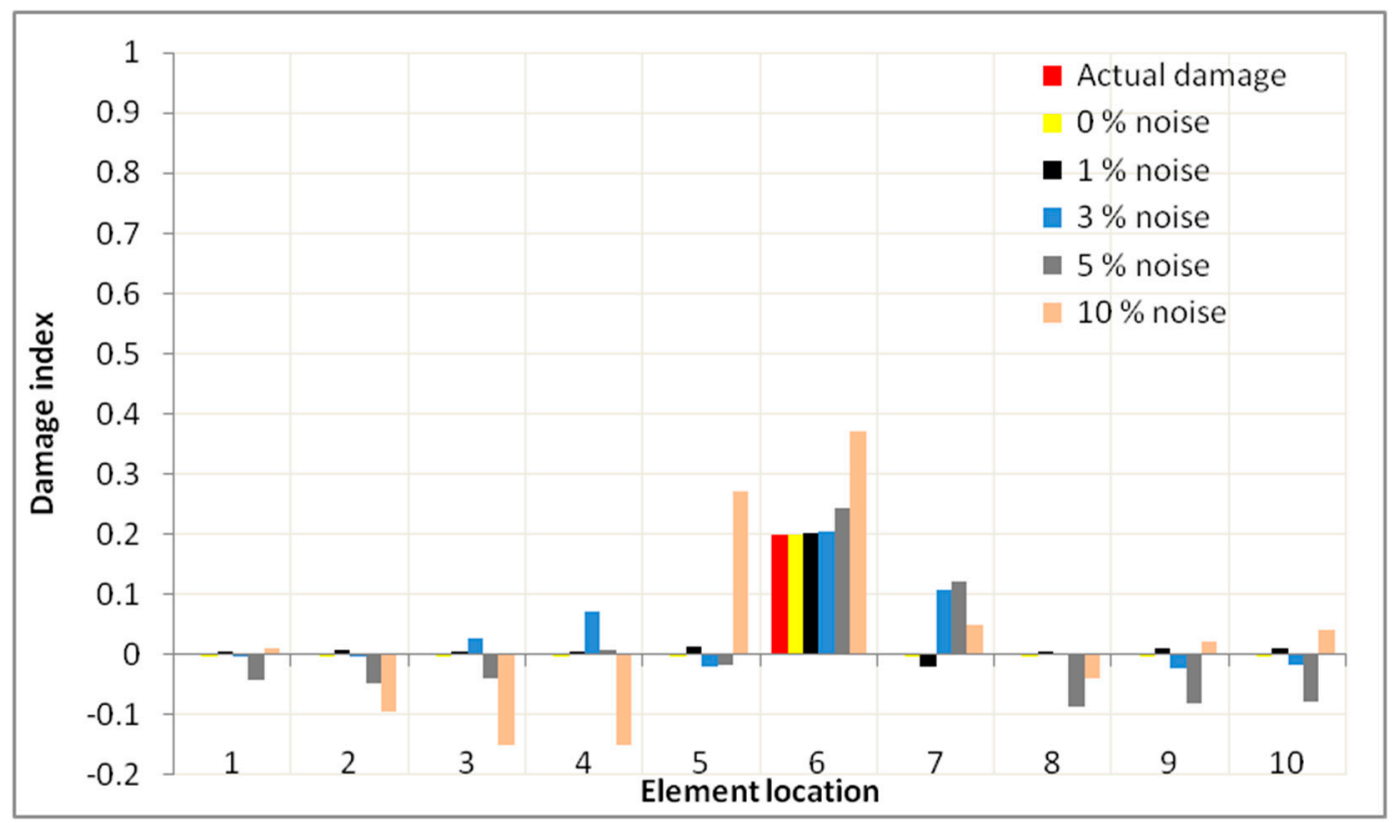

(a)

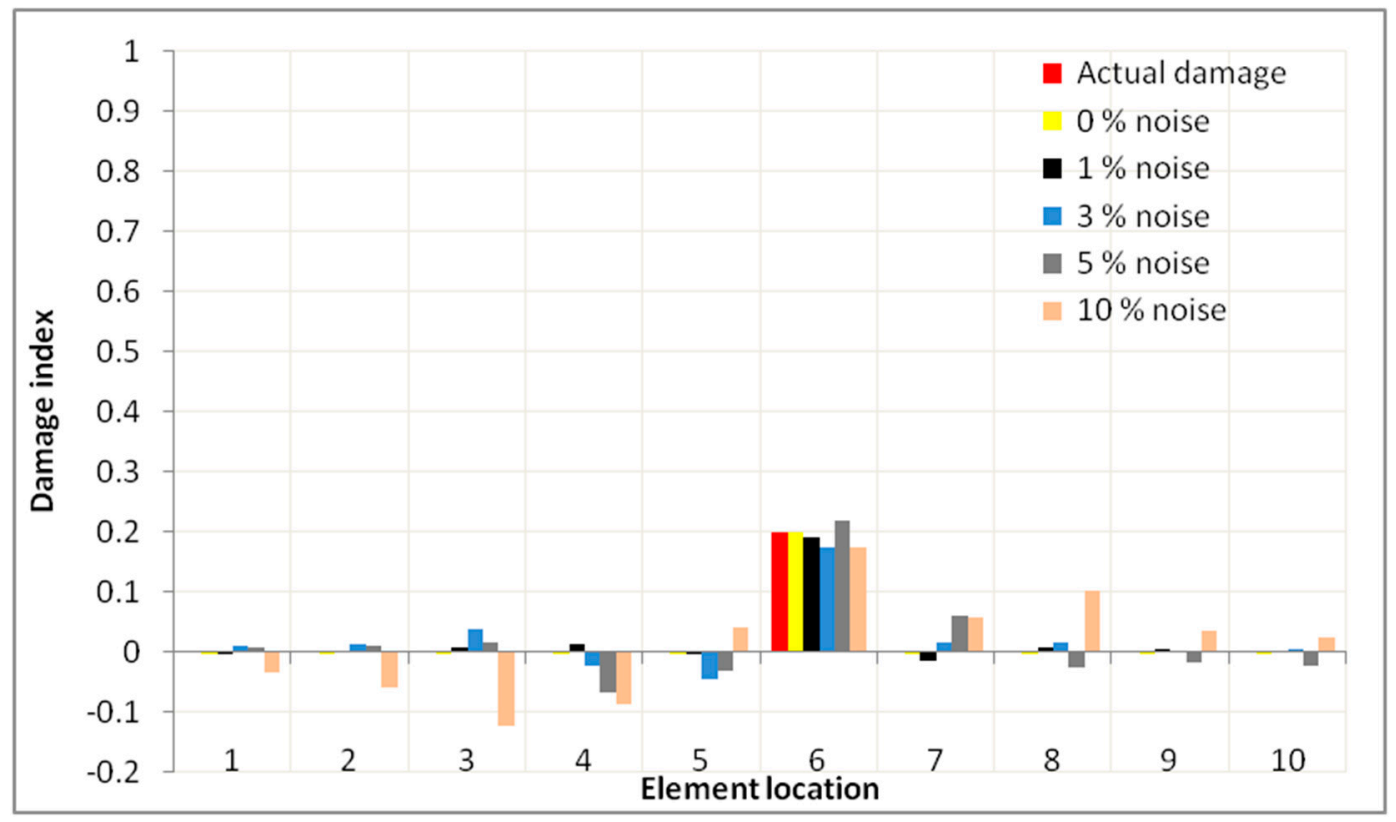

(b)

Figure 2. Damage distribution for Case 1 using Objective Function I for (a) noise both in frequency and mode shapes and (b) noise in mode shapes only.

The results of Objective Function I are discussed further here (Figure 2). For different noise levels, the damage index is detected to be very near the actual damage index of 0.2 at $0 \%, 1 \%$ and $3 \%$ noise (Figure 2a,b). However, for noise at $5 \%$ in both frequencies and mode shapes (Figure 2a), the damage index was 0.24 , which further degraded to 0.37 for $10 \%$ noise levels. Similarly, the damage index for noise in mode shapes only (Figure $2 b$ ) degraded to 0.22 and 0.17 as compared to the actual damage index of 0.2 in the case of $5 \%$ and $10 \%$ noise, respectively. It can be noticed that less degradation is seen in the mode shape noise than noise in both frequencies and mode shapes, which is rational. Likewise erroneous detection of damage in other elements especially close to damage elements is also more evident in noise in both the frequencies and mode shapes case than the noise in mode shapes only. 


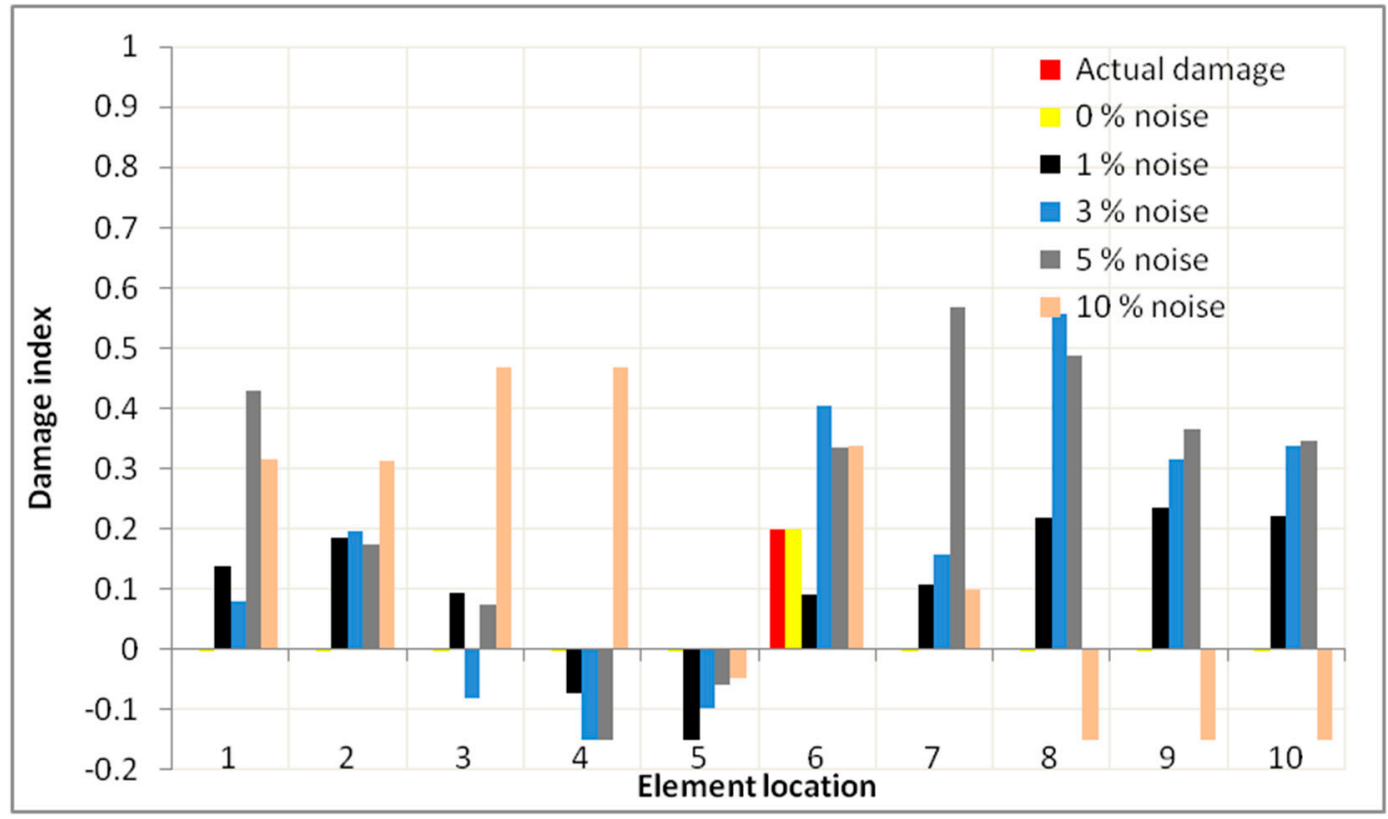

(a)



(b)

Figure 3. Damage distribution for Case 1 using Objective Function II for (a) noise both in frequency and mode shapes and (b) noise in mode shapes only.

It can be noted that all the objective functions have similar frequency-related residual included in their first part (see Equation (11)). Therefore, the differences in the results can be mainly attributed to the second part of the objective functions, and possible reasons related to the performance of each of the objective function are discussed now. 


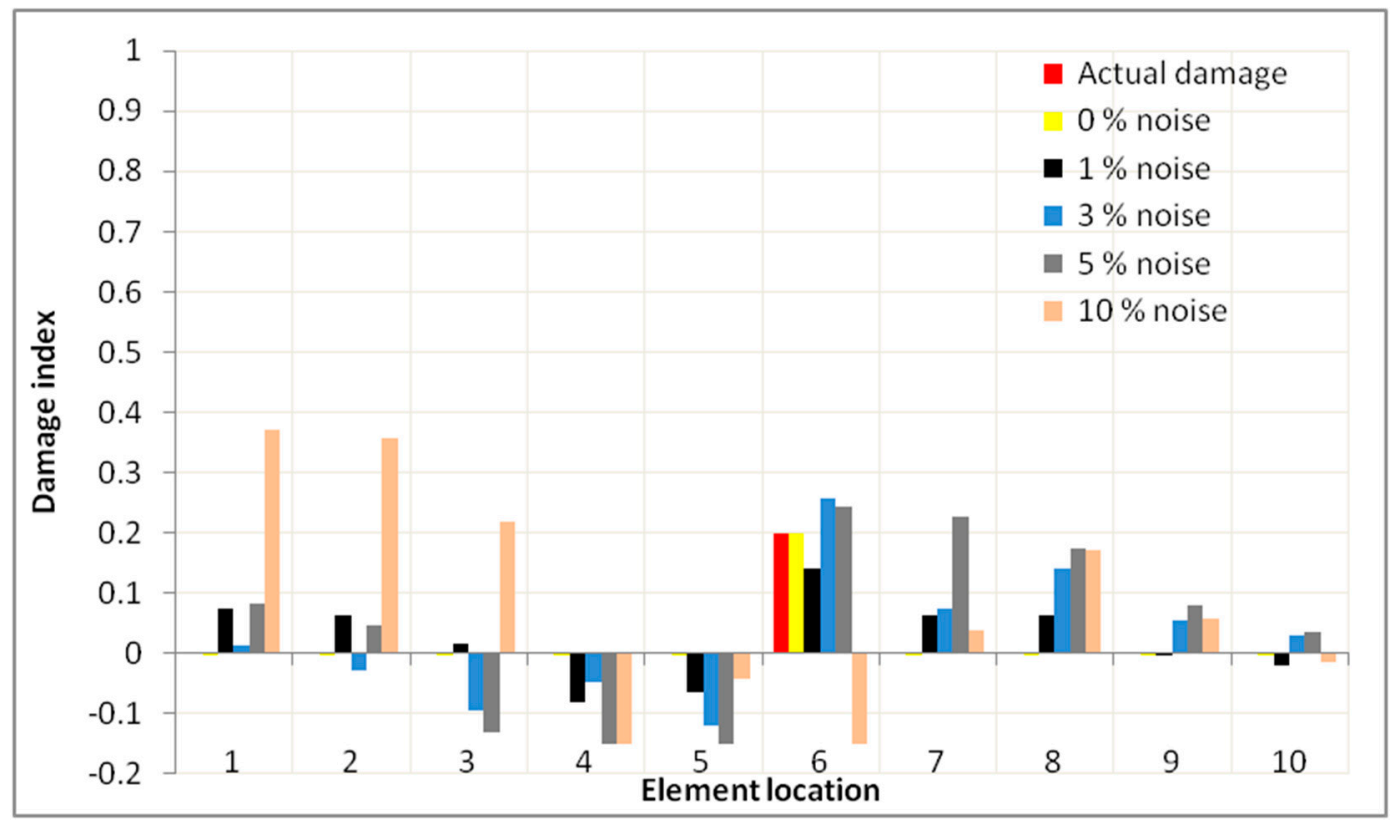

(a)



(b)

Figure 4. Damage distribution for Case 1 using Objective Function III for (a) noise both in frequency and mode shapes and (b) noise in mode shapes only.

The Objective Function I is based on the MAC value, which is a basically a statistical indicator between two mode shapes. A single value for a particular mode shape near one indicates perfect correlation. The MAC value has been shown to be more sensitive to large difference in mode shapes as compared to small differences [49]. Furthermore, it has also been noted that the MAC value is less sensitive to noise as it tries to average out the values of mode shapes for a particular mode. However, Objective Function II is mainly related to modal flexibility. The coefficients of the modal flexibility matrix have been recognized as being very sensitive to damage [50]. The error gets amplified as the values of frequencies and mode shapes having noise get multiplied with each other. Thus, 
although it is a good detector of the relative damage between the elements, it may also result in large numerical errors at elements where damage is not present. Lastly, Objective Function III related to strain energy also amplifies the errors as the expanded noisy mode shape gets multiplied with the stiffness matrix to calculate the strain energy. This discussion could be further associated with the objective function values obtained for each case. For example, the objective function values obtained for $10 \%$ noise in frequencies and mode shapes for all the objective functions are discussed here for comparison. The objective function values were $1 \times 10^{-5}, 1.03$ and 0.004 for Objective Functions I, II and III, respectively, for $10 \%$ noise in frequencies and mode shapes. It can be seen that the minimum value has been obtained for Objective Function I and the maximum value for Objective Function II. This is also consistent with the damage indices obtained in Figures 2-4. As a result, the sparseness of the results with respect to different elements is more for Objective Functions II and III as compared to Objective Function I. Therefore MAC-based Objective Function I presents itself as a good candidate for damage detection purposes due to less sparseness in the results.

Due to wrong detections with different objective functions especially in noisy conditions, a suitable technique needs to be applied to address the damage detection issue in non-damaged elements. A sensitivity analysis of all the elements was performed. It has been revealed that elements closer to the supports have very low sensitivities. Such elements can cause ill-conditioning of the problem, and a small change in the value results in a large change in the modal data. Furthermore, elements at equal distance from the center of the beam have equal sensitivities, e.g., Element Nos. 5 and 6 influence the modal data in a similar manner. Such elements can also lead to similar modal values. Regularization techniques in the context of model updating have been applied by various researchers using SM. SM has the drawback of being stuck in local minima and involves finding the inverse of the sensitivity matrix. The choice of suitable regularization parameter was also based on the sensitivity matrix, and corresponding filter factors have been calculated using techniques such as GCV, L-curve or quasi-optimality functions [37]. However, in the context of this paper, a regularization technique was applied using GA to harness the benefits of both techniques. The method adopted in this study is a forward analysis method, which does not involve solving the inverse of matrices. Furthermore, EAs have a limitation that they work on a number of points in parallel contrary to SM-based methods, which work on a single point at a time. Therefore, in this paper, based on the basic concept of the L-curve, Pareto fronts using multiobjective optimization were employed, as these can be applied graphically in the context of EAs. The tradeoff value can be selected where the curvatures of the three corresponding points change with maximum bend angle [25].

Therefore, the Pareto optimal front has been used in this study to find out the tradeoff between the objective function and regularization part as explained in Equation (15). Multiobjective GA implemented in MATLAB using function gamultiobj has been used. A population size of 100, maximum generation limit of 1000 and function tolerance of $1 \times 10^{-10}$ havebeen used for the multiobjective function. A typical Pareto front was obtained for Damaged Beam 1 and is shown in Figure 5 for $5 \%$ noise in frequencies and mode shapes. It has been found that a value of $6 \times 10^{-6}$ gives a suitable tradeoff between the two axes. Likewise, other tradeoff points have been selected for other noise levels based on their corresponding Pareto fronts and are reported in Table 1. It can be seen that the value of these parameter decreases with low noise and increases with high noise. Likewise for noise in mode shapes only, the values of these parameters are generally less than noise both in frequencies and mode shapes. This is logical as the parameter essentially is a penalty function, which weights the need for inclusion of the function $\left\|x-x_{o}\right\|$ in the overall objective function value. The more the noise, the more will be the tradeoff value. 


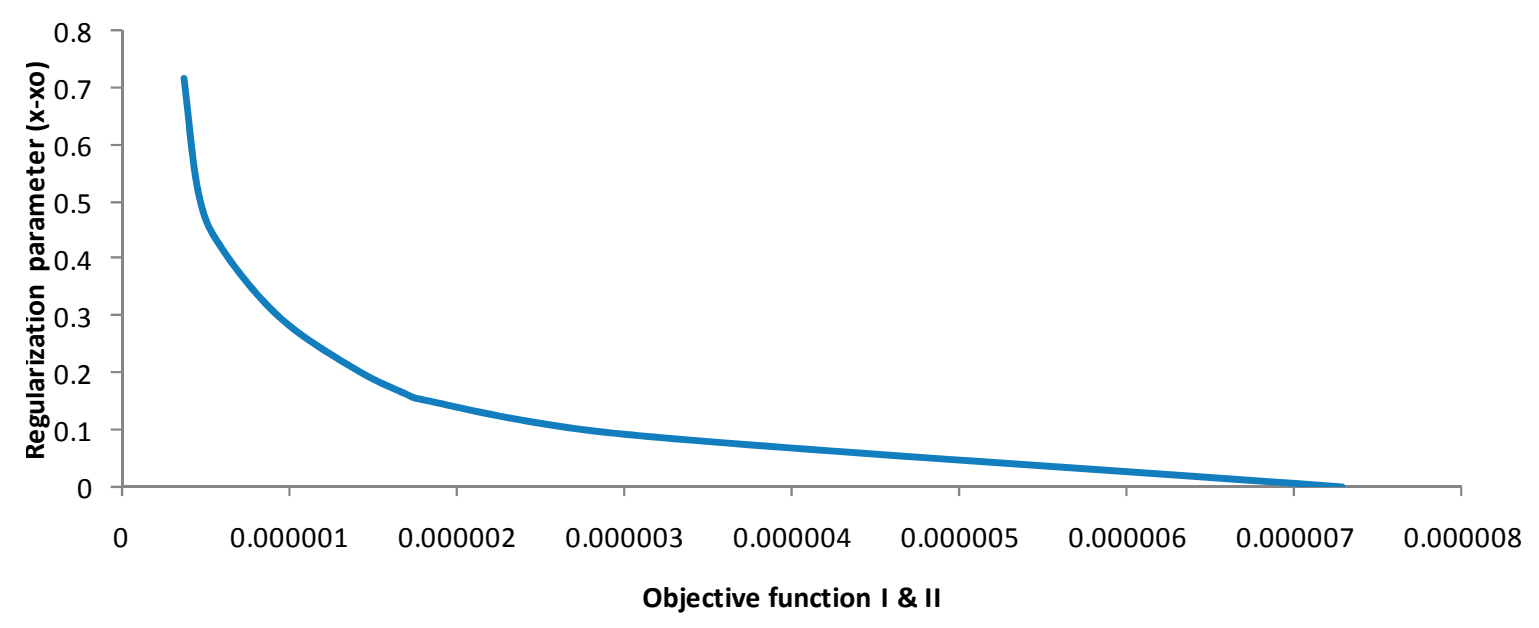

Figure 5. Typical Pareto front for Case 1 using Objective Function I with 5\% noise both in frequencies and mode shapes.

Table 1. Tradeoff value for Case 1 for all noises cases.

\begin{tabular}{cccc}
\hline \multirow{2}{*}{ Serial No. } & \multicolumn{2}{c}{ Noise in } & \multirow{2}{*}{ Tradeoff Value } \\
\cline { 2 - 3 } & Frequencies (\%) & Mode Shapes (\%) & \\
\hline 1 & 0 & 0 & - \\
2 & 1 & 1 & $1 \times 10^{-7}$ \\
3 & 3 & 3 & $9 \times 10^{-6}$ \\
4 & 5 & 5 & $6 \times 10^{-6}$ \\
5 & 10 & 10 & $3 \times 10^{-4}$ \\
6 & 0 & 1 & $1 \times 10^{-10}$ \\
7 & 0 & 3 & $7 \times 10^{-7}$ \\
8 & 0 & 5 & $6 \times 10^{-7}$ \\
9 & 0 & 10 & $4 \times 10^{-6}$ \\
\hline
\end{tabular}

GA was applied using Equation (15) with Objective Function I, II and III. The effect of regularization on all the objective functions is shown in Figures 6-8. It can be seen that Objective Function I has shown promising results as compared to the other two objective functions. This advocates that noise errors cannot be eliminated through regularization without a proper objective function. Figure 6 shows the damage index of the updated damaged model for all noise levels for regularized Objective Function I. For the damaged beam, the damage index for Element No. 6 for the regularized objective function was found to be very close to the actual value of 0.2 in most cases; e.g., for $10 \%$ noise in frequencies and mode shapes, the damage index is found to be 0.24 as compared to the actual damage index of 0.2 (Figure 6a). Likewise, for $10 \%$ noise in mode shapes, the damage index for Element No.6 turns out to be 0.14 (Figure 6b). Likewise, the wrong detections in other elements are also much less with the maximum value at -0.028 for $10 \%$ noise in frequencies and mode shapes. It can be noted from the damage indices that regularized model updating has shown promising results. 


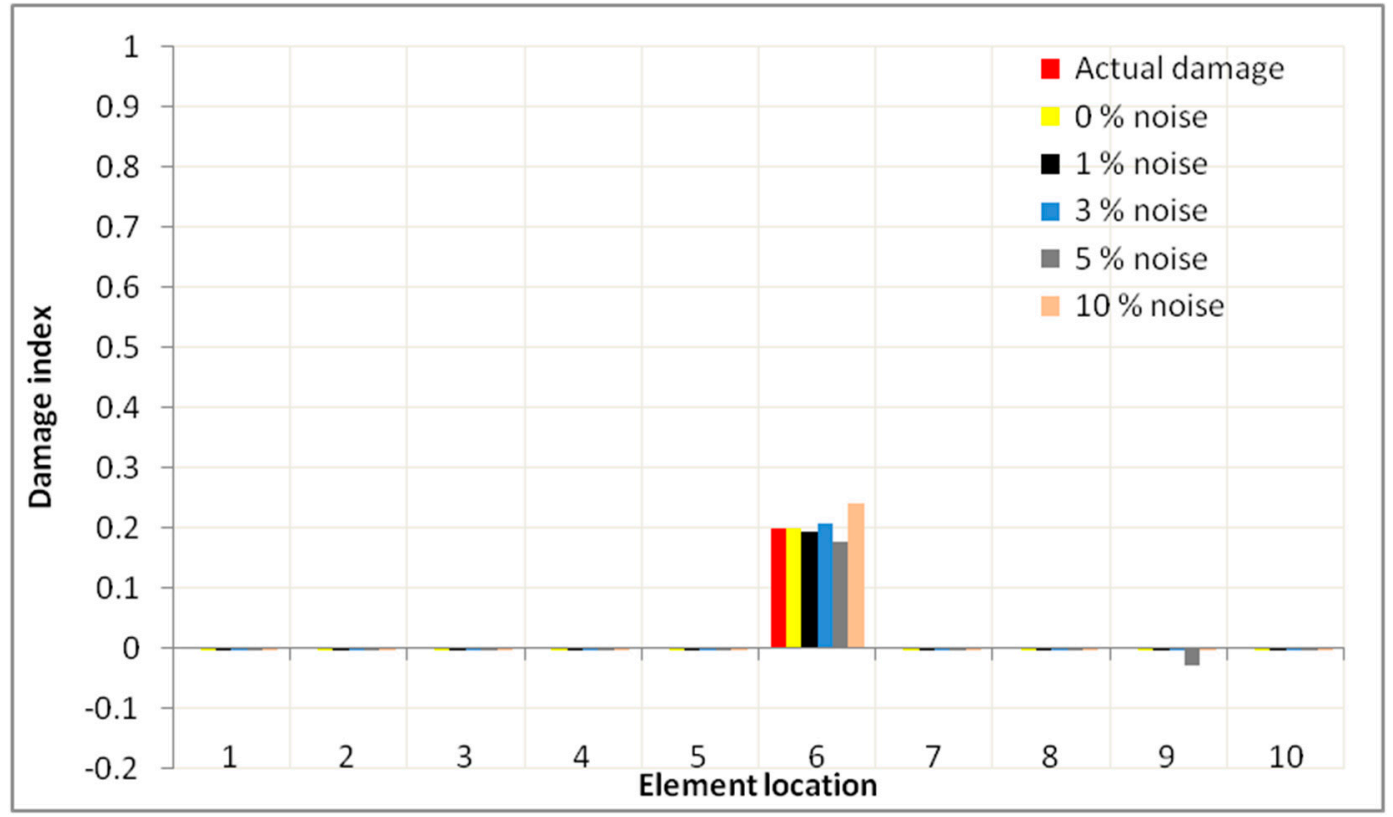

(a)

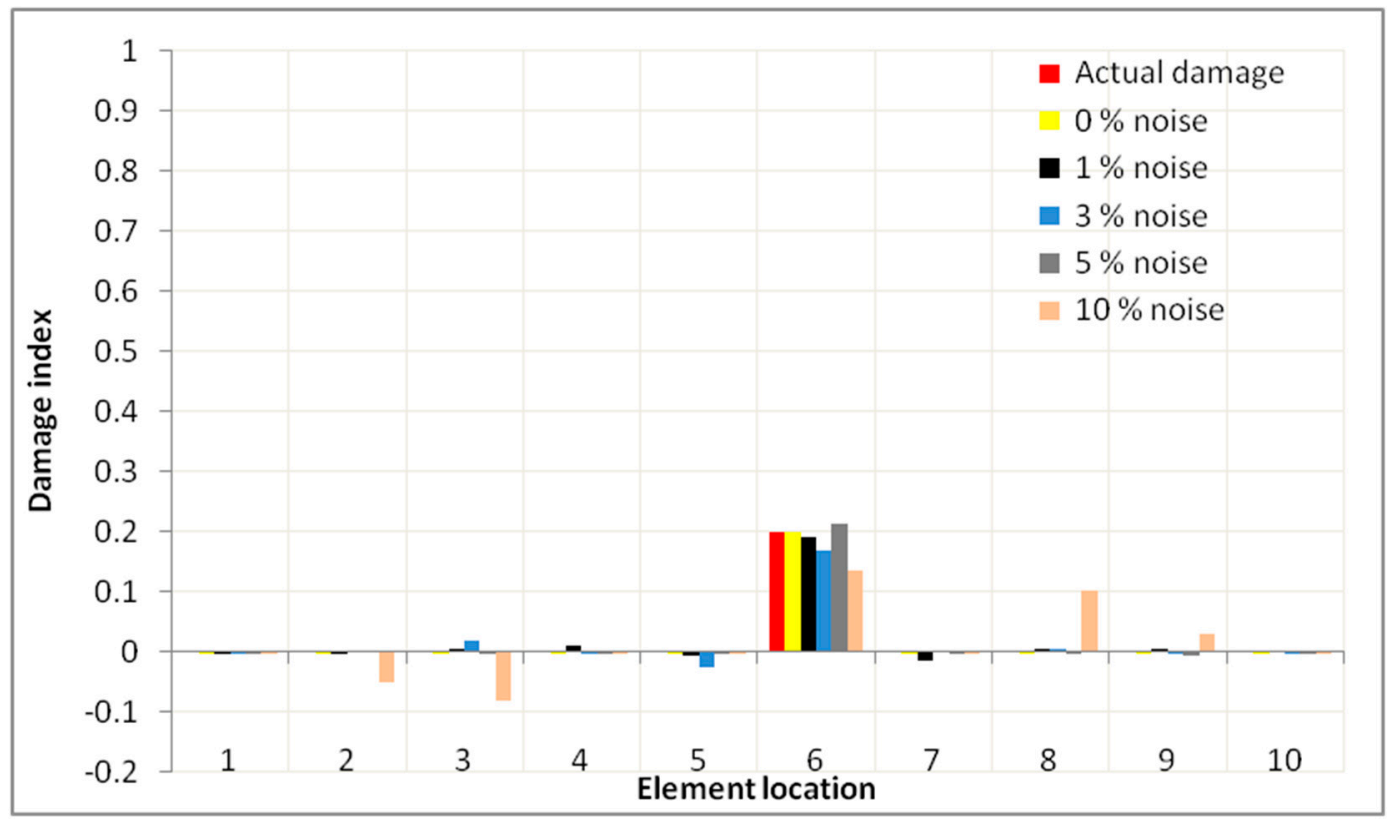

(b)

Figure 6. Damage distribution for Case 1 using Objective Function I with regularization for (a) noise both in frequency and mode shapes and (b) noise in mode shapes only.

This proves that the proposed approach is more efficient in updating. The regularization has improved the conditioning of the problem and thus leads to physically justifiable solutions. However, this regularization has assumed that the initial estimate of the model, i.e., $\mathrm{x}^{0}$ is considered as a good representative of the actual structure. This assumption may not be valid in all the cases where a priori model realization may be significantly different from the actual physical structure. However, the results advocate that the proposed method utilizes the benefits of both EAs, as well as regularization function simultaneously. 


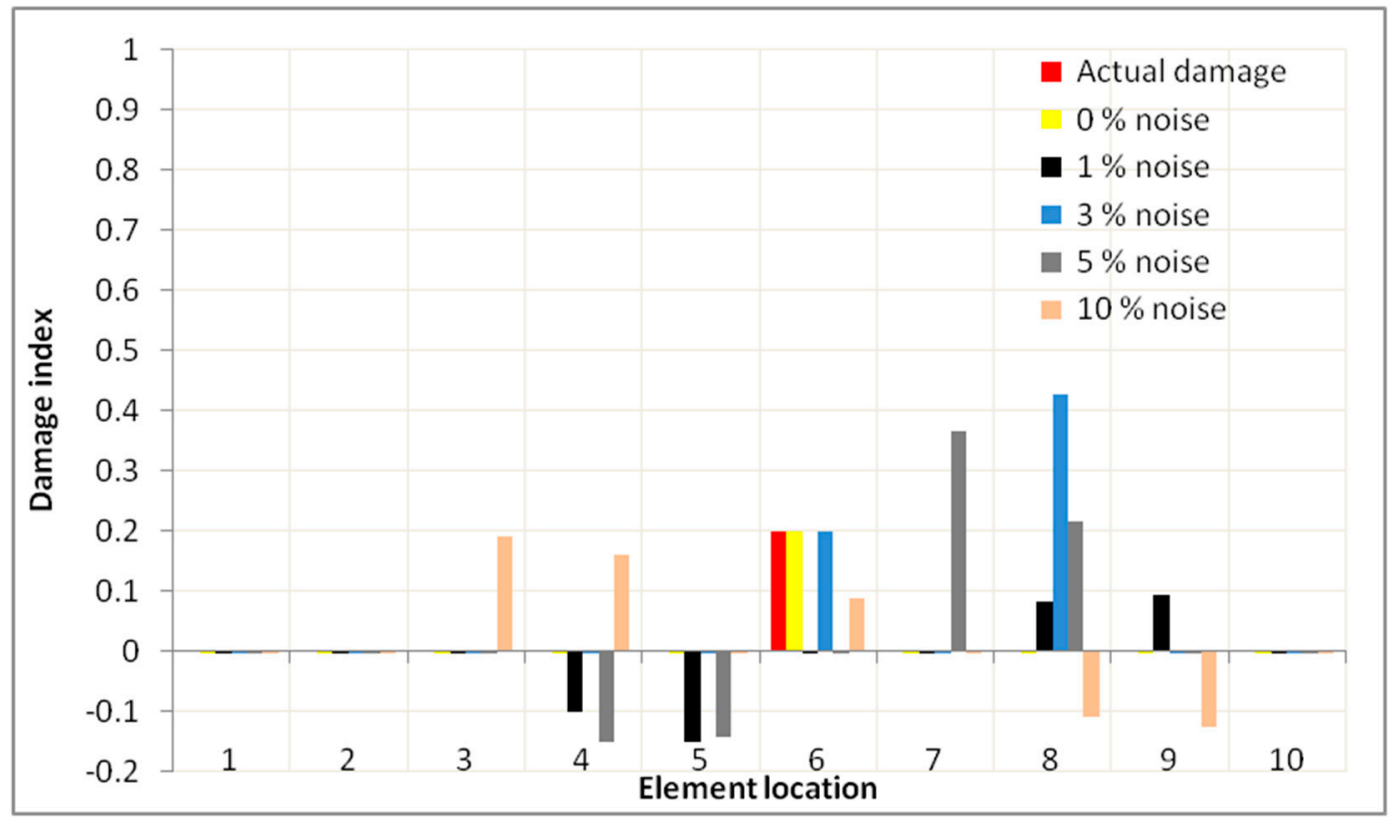

(a)

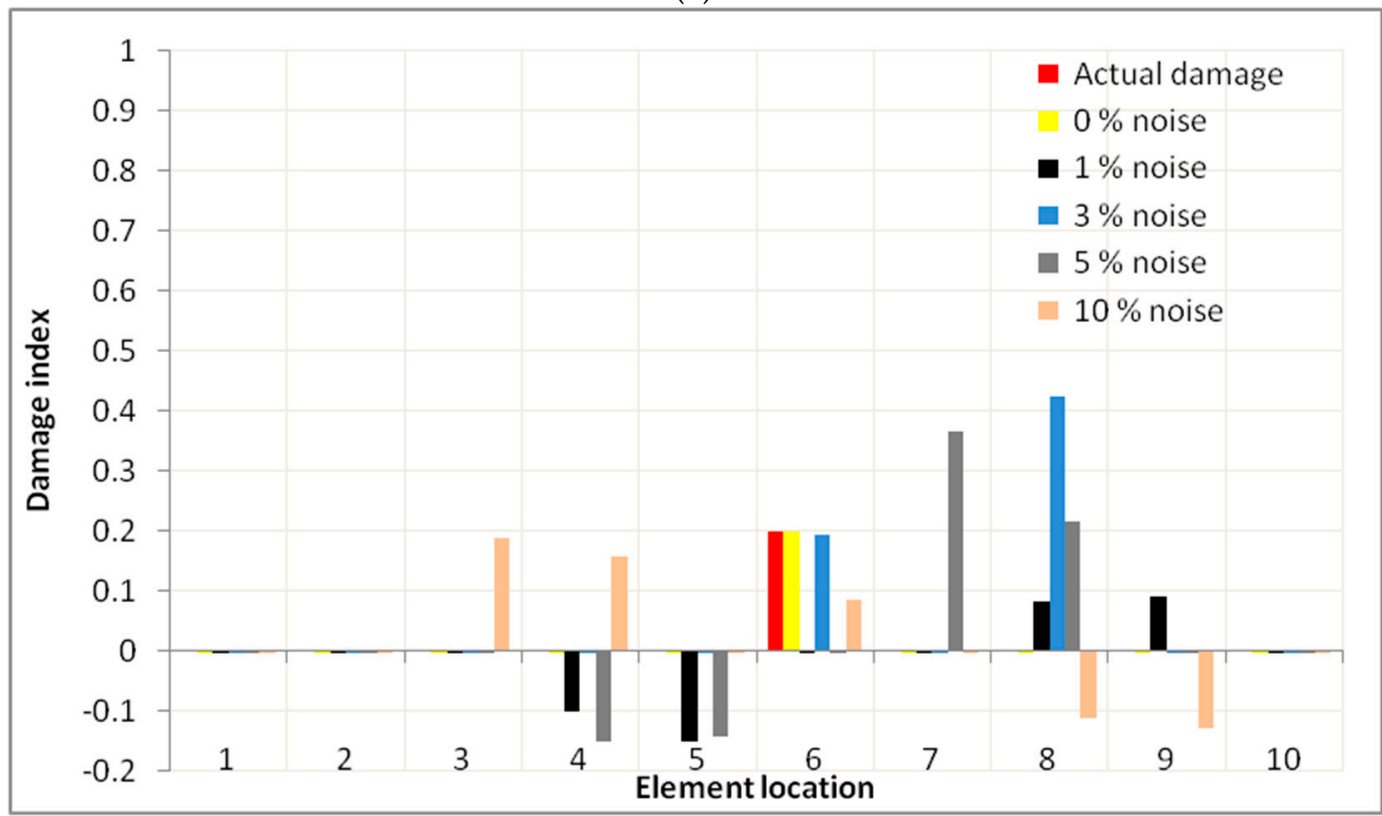

(b)

Figure 7. Damage distribution for Case 1 using Objective Function II with regularization for (a) noise both in frequency and mode shapes and (b) noise in mode shapes only.

\subsection{Simulated Simply Supported Beam 2 (Case 2)}

In this case (Case 2), reduction of MOI of multiple elements has been considered to check the effectiveness of the proposed approach. All the other properties of the beam match with Case 1. The damage was introduced in the $3 \mathrm{rd}$, 6th and 9th element as $30 \%, 50 \%$ and $20 \%$, respectively, in the form of reduction in MOI as compared to the reference model, which corresponds to a damage index of $0.3,0.5$ and 0.2. The location near the support has been selected as it is considered tough to detect damage located near the supports. GA was again used to minimize the three objective functions. The first three mode shapes were considered. 


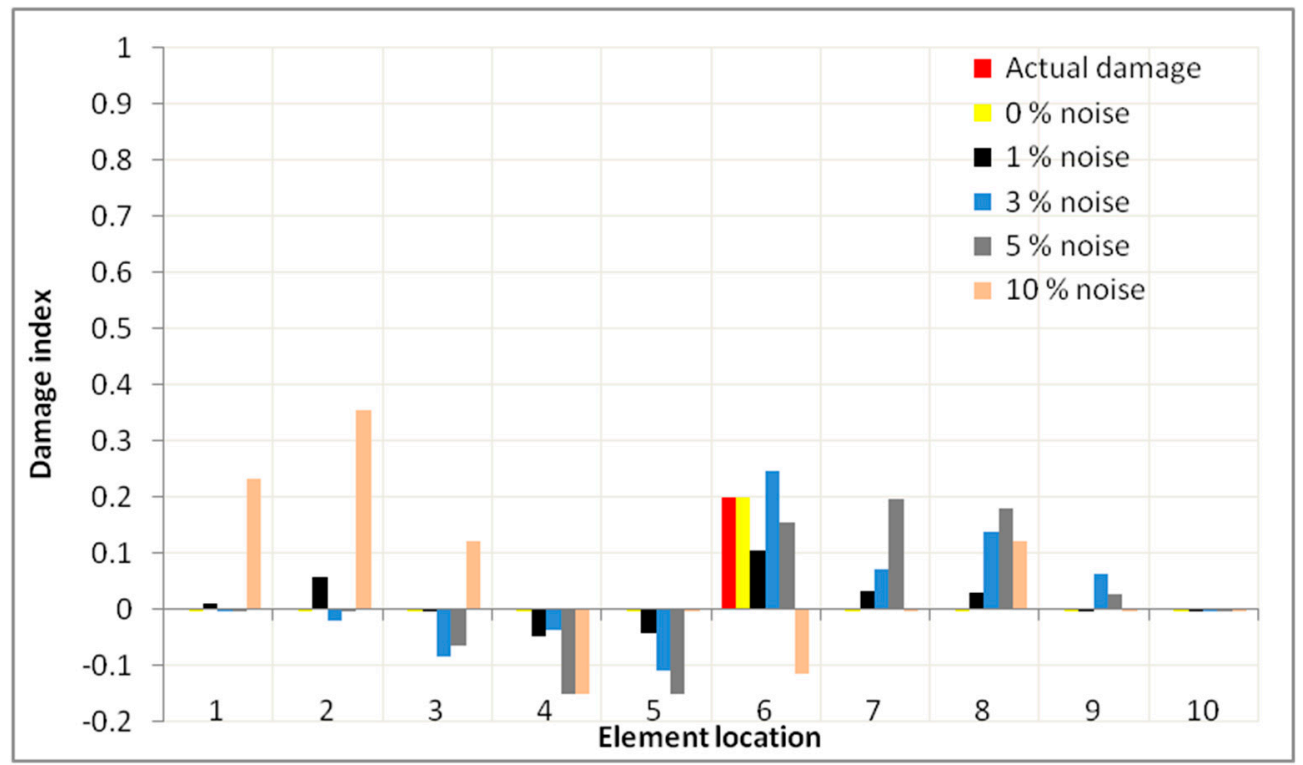

(a)

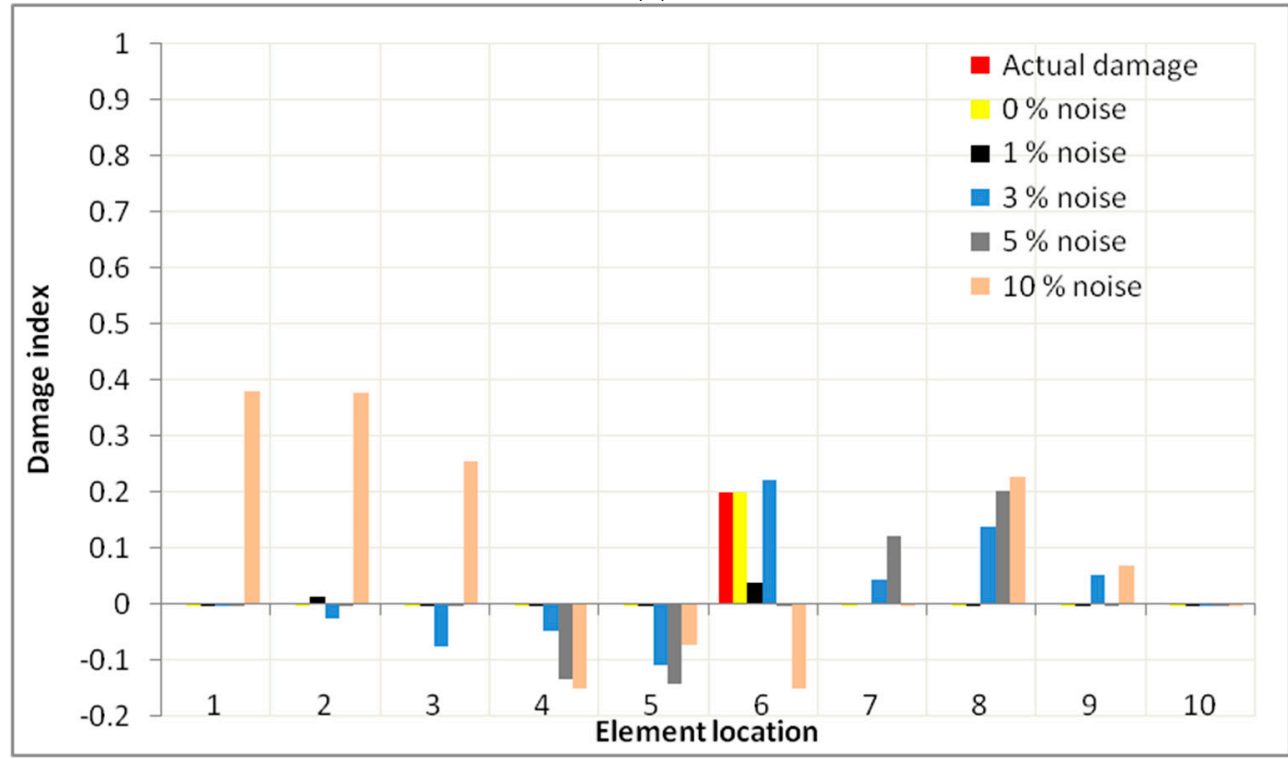

(b)

Figure 8. Damage distribution for Case 1 using Objective Function III with regularization for (a) noise both in frequency and mode shapes and (b) noise in mode shapes only.

A similar technique as used earlier for Case 1 was used for assessment of the damage severity. The damage indices were calculated first by applying the three objective functions, and the results are presented in Figures 9-11 in a similar pattern as in Case 1. At $0 \%$ noise levels, all the objective functions performed well. However, it was noticed that Objective Function I performed better than the other two in noisy conditions. For $10 \%$ noise in frequencies and mode shapes, the damage index for the three Damaged Element Nos. 3, 6 and 9 was found to be 0.31, 0.52 and 0.11, respectively (actual damage index $=0.3,0.5$ and 0.2 ), for Objective function 1 ; whereas, the corresponding values for these elements were $0.36,0.28$ and 0.09 for Objective Function II and $0.65,0.25$ and 0.18 for Objective Function III. It can be seen that Objective Function I gave the best results although some wrong detections were also present. 


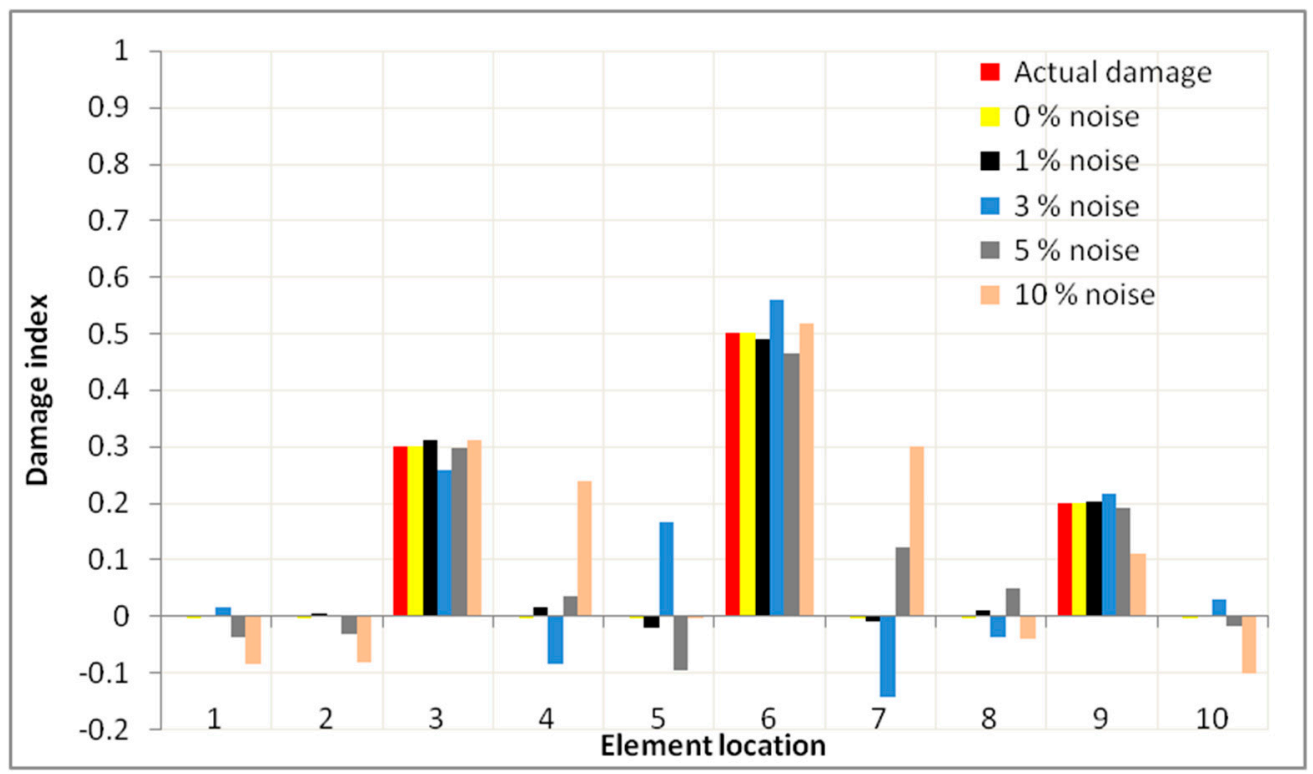

(a)

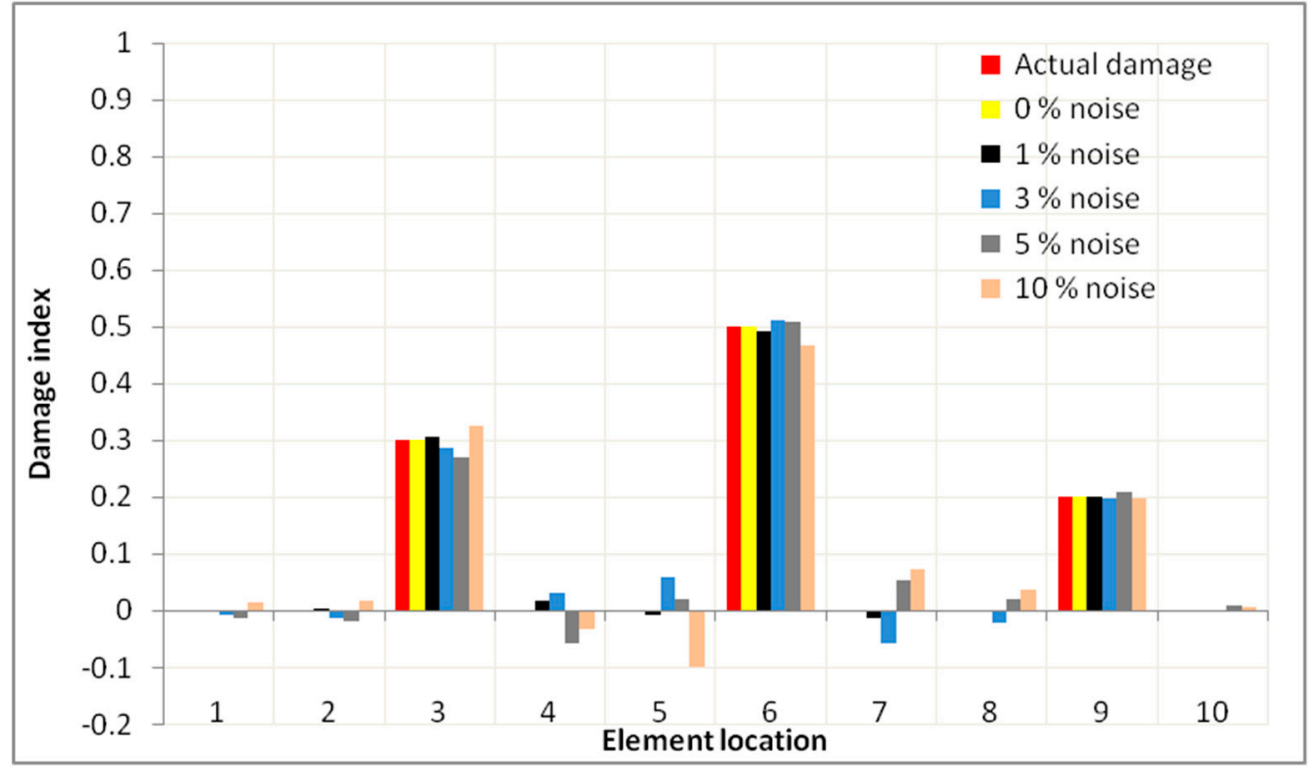

(b)

Figure 9. Damage distribution for Case 2 using Objective Function I for (a) noise both in frequency and mode shapes and (b) noise in mode shapes only.

The Objective Function I was then regularized using Equation (15). The damage severity indices are presented in Figures 12-14 for all three objective functions. As observed in Case 1, the regularization has been effective for Objective Function I as compared to Objective Functions II and III. From the results in Figure 12 for Objective Function I, it can be seen in all the cases that damage estimation from the regularization technique is more accurate than when the Objective Functions I, II and III were applied without regularization in noisy conditions. For $10 \%$ noise, the damage index for the three Damaged Element Nos. 3, 6 and 9 was found to be 0.28, 0.52 and 0.07, respectively (actual damage index $=0.3,0.5$ and 0.2 ). It was also found that the regularized updating has good detection of undamaged elements when compared to the un-regularized case. Furthermore, the detection was satisfactory even in the worst case conditions, i.e., at $10 \%$ noise. 


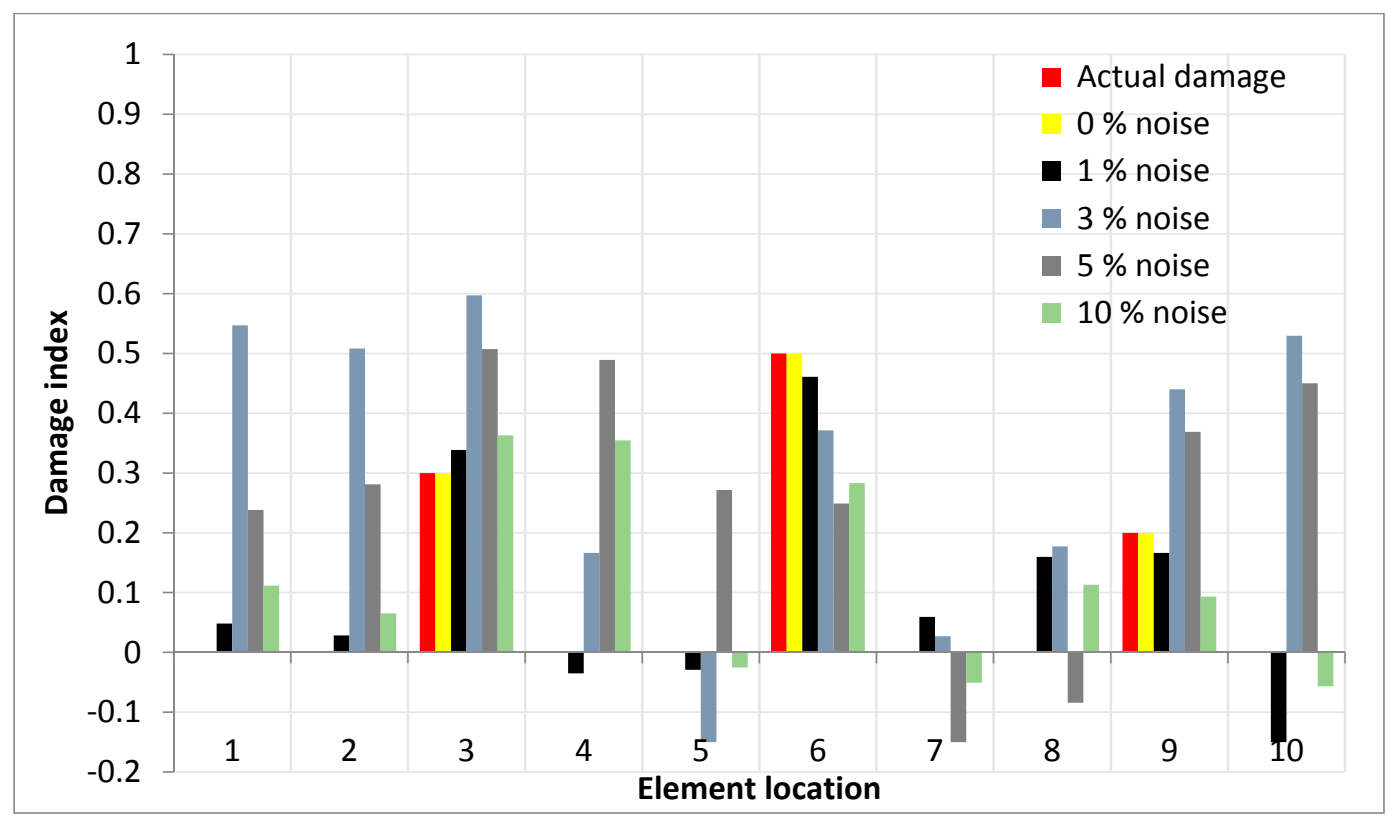

(a)

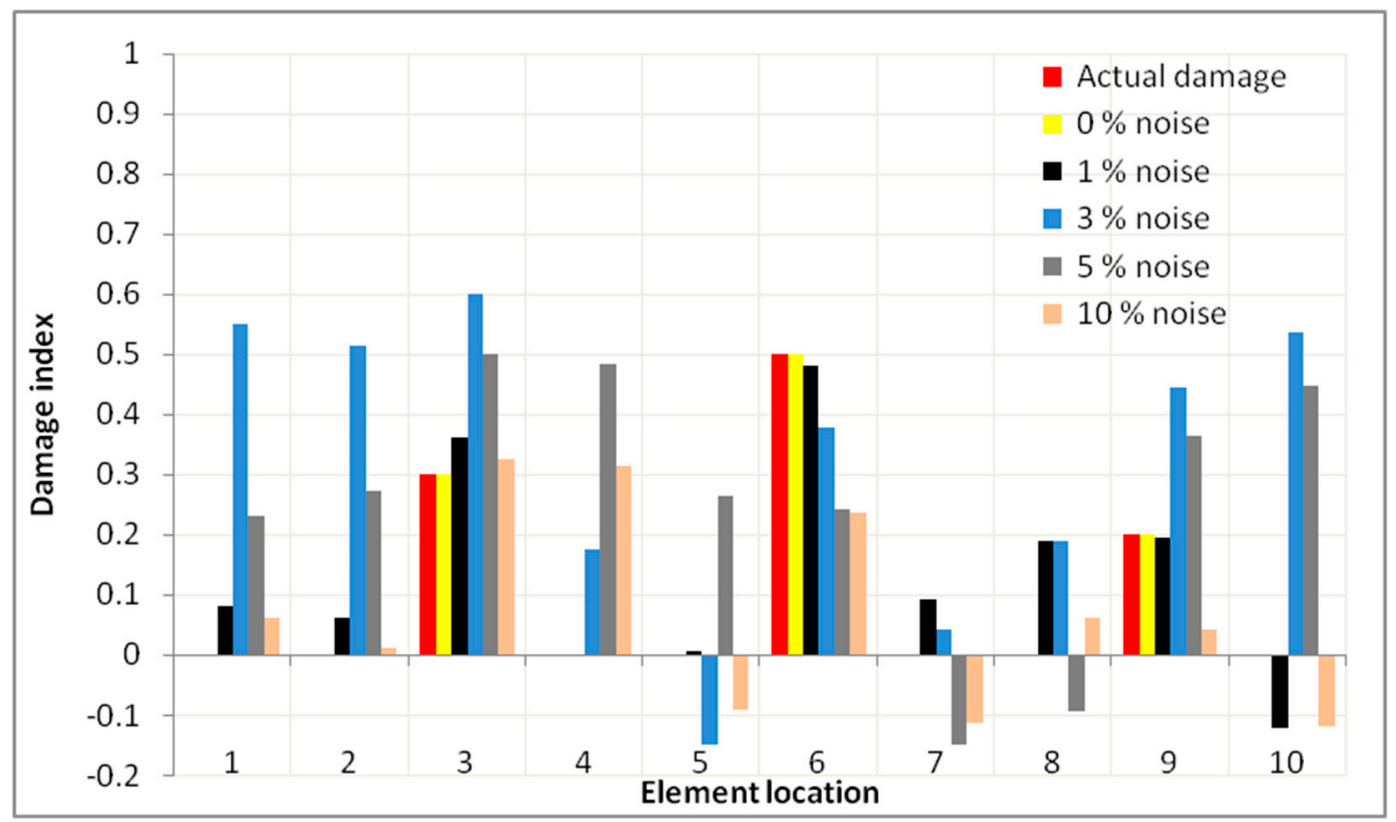

(b)

Figure 10. Damage distribution for Case 2 using Objective Function II for (a) noise both in frequency and mode shapes and (b) noise in mode shapes only.

\subsection{Experimental Beam (Case 3)}

In actual structures, there are differences in the measured quantities as compared to their theoretical counterparts mainly due to differences in material and geometrical properties. Damage detection becomes more difficult in these problems, and it is imperative to check the effectiveness of the proposed approach on actual experimental test. Furthermore, by using this example, comparative analysis can be easily made with other studies.

The experimental beam was taken from $\mathrm{Hu}$ et al. [45]. The aluminum beam has a rectangular section of $0.05 \mathrm{~m} \times 0.006 \mathrm{~m}$ with two fixed ends. The length of the beam is $0.6 \mathrm{~m}$. Other material 
specifications were the modulus of elasticity as $70 \mathrm{GPa}$, the Poisson's ratio as 0.3 and the density as $2700 \mathrm{~kg} / \mathrm{m}^{3}$. A saw cut damage was induced in the beam at the ninth element by cutting a quarter of the total thickness from the top and bottom surface of the intact specimen. The theoretical damage index for the cracked location was 0.875 , i.e., an $87.5 \%$ reduction of $\mathrm{MOI}$ in y direction. The first three mode shapes were compared as these were measured experimentally.

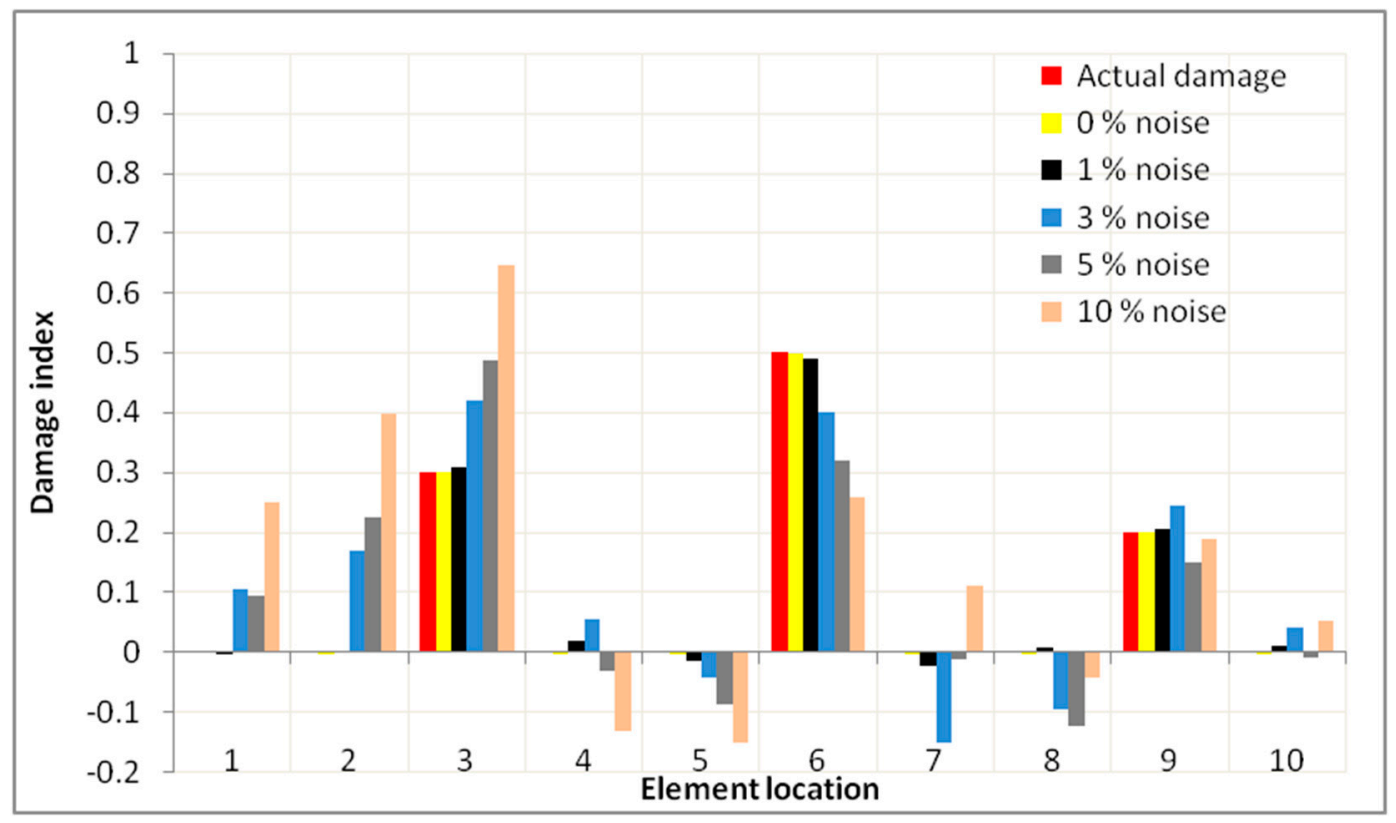

(a)



(b)

Figure 11. Damage distribution for Case 2 using Objective Function III for (a) noise both in frequency and mode shapes and (b) noise in mode shapes only. 


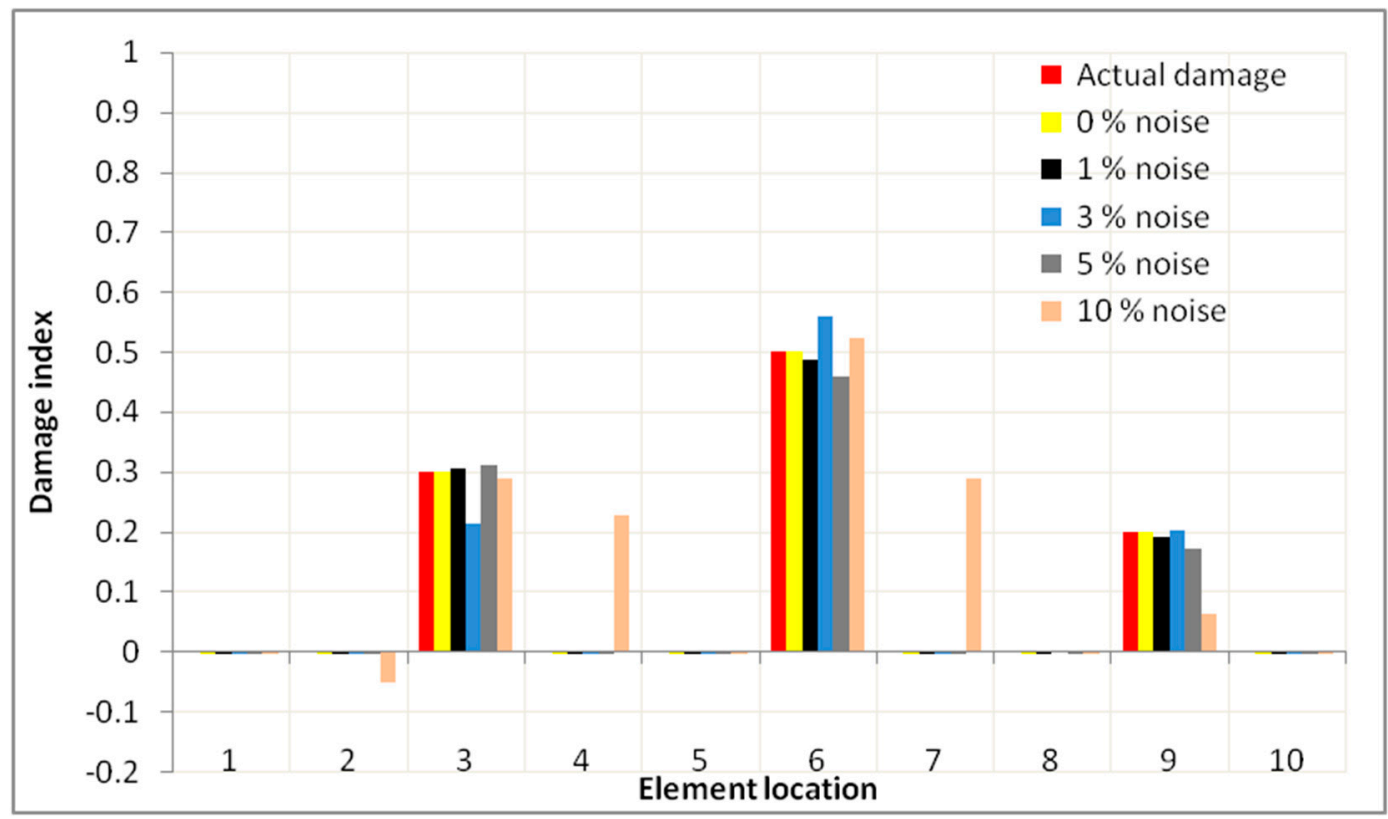

(a)

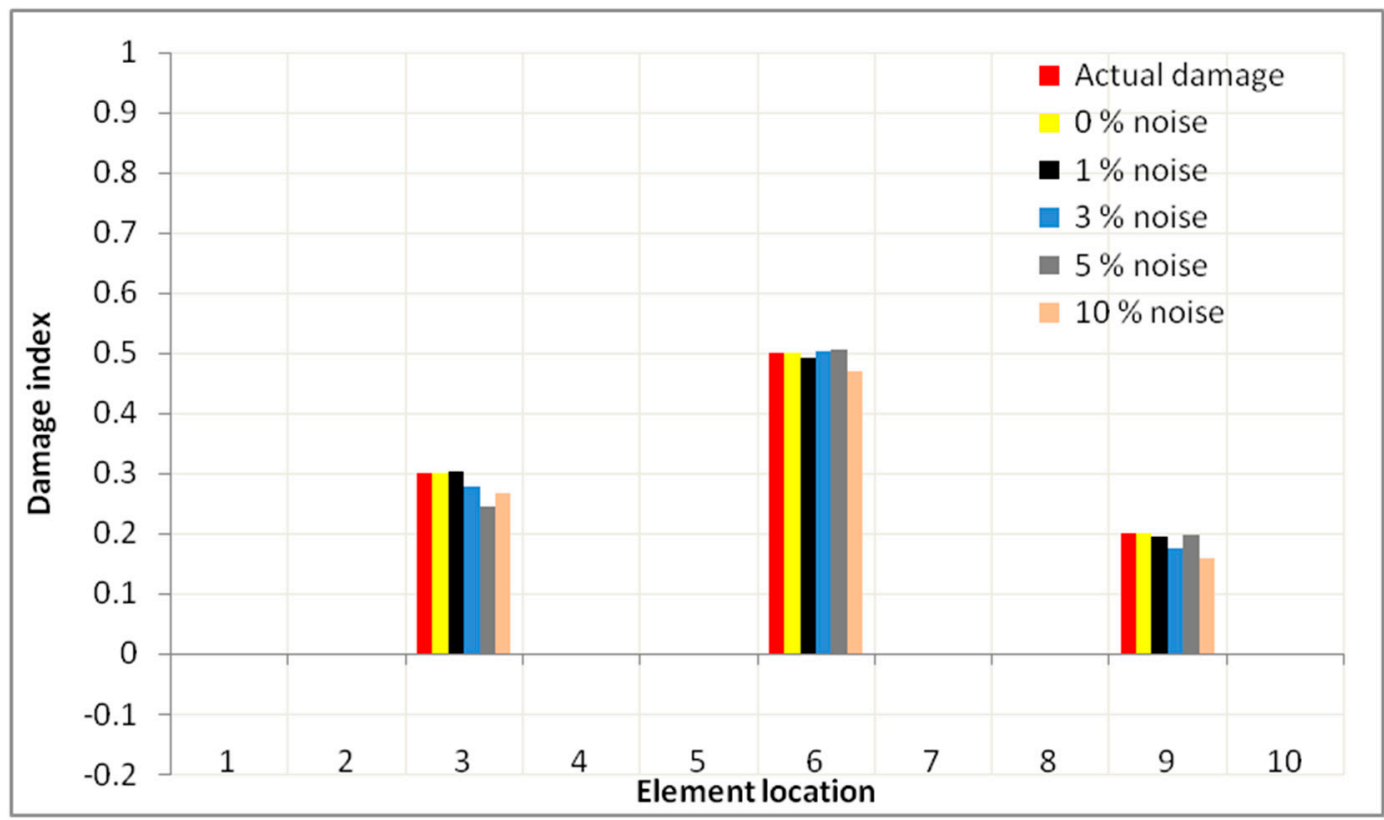

(b)

Figure 12. Damage distribution for Case 2 using Objective Function Iwith regularization for (a) noise both in frequency and mode shapes and (b) noise in mode shapes only.

SAP 2000 was used to model and analyze the beam. For the experimental beam, the adopted mesh has been selected based on convergence analysis of the FE model. For this purpose, the beam was divided into 10, 20 and 40 nodes having a thickness of $0.5 \mathrm{~cm}$, and the mesh convergence effect has been checked based on the first three natural frequencies. The \% error between the 10 - and 20-node beam was $0.0034,0.0058$ and $0.1641 \%$, whereas the error between the 20 - and 40 -node beam was $0.0000,0.0016$ and 0.0070 for the first three frequencies respectively. Therefore, due to the maximum error of $0.1641 \%$ between 10 and 20 nodes and $0.0070 \%$ between 20 and 40 nodes, the beam with 20 nodes has been selected. The beam with 20 equal two-dimensional beam elements is also 
consistent with the measured DOF in the experiment. Fixed end supports were imposed on the corner elements of the beam. The mass and stiffness matrices were afterwards exported to MATLAB for optimization purposes.



(a)



(b)

Figure 13. Damage distribution for Case 2 using Objective Function II with regularization for (a) noise both in frequency and mode shapes and (b) noise in mode shapes only.

The model was updated considering the first three natural frequencies and mode shapes for damage detection. The three objective functions are applied to update the beam to check the suitability of the objective functions in the actual test. The damage indices identified by using Objective Functions I, II and II are shown in Figure 15a. It can be noticed that for this damage case, the damage 
index for Element No. 9 (actual damage index $=0.875$ ) was found to be $0.55,0.65$ and 0.61 for Objective Functions I, II and III, respectively. However, as compared to Objective Function I, misdetection in other elements was of much greater magnitude in Objectives II and III. The results are also consistent with the earlier results of Case 1 and Case 2.

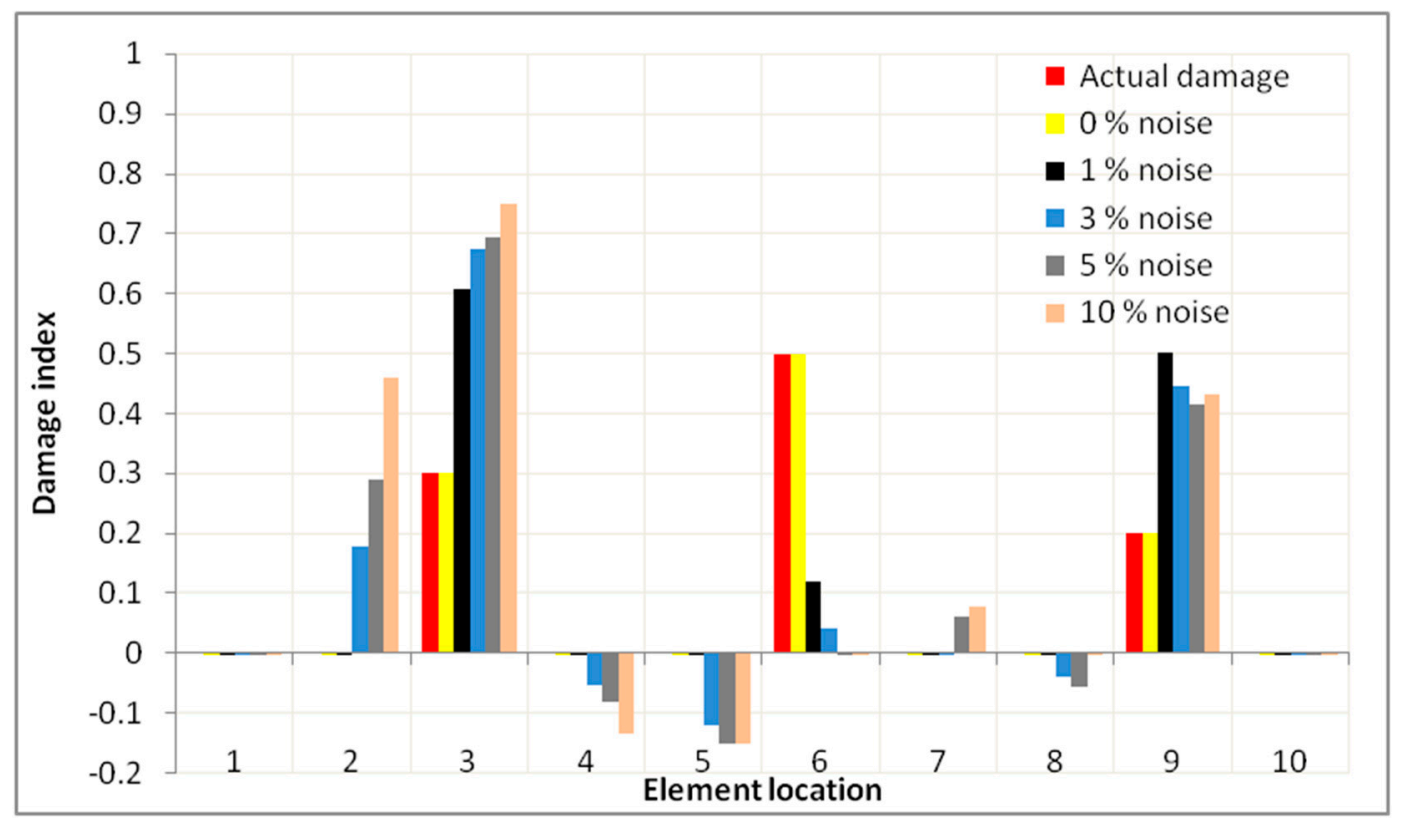

(a)

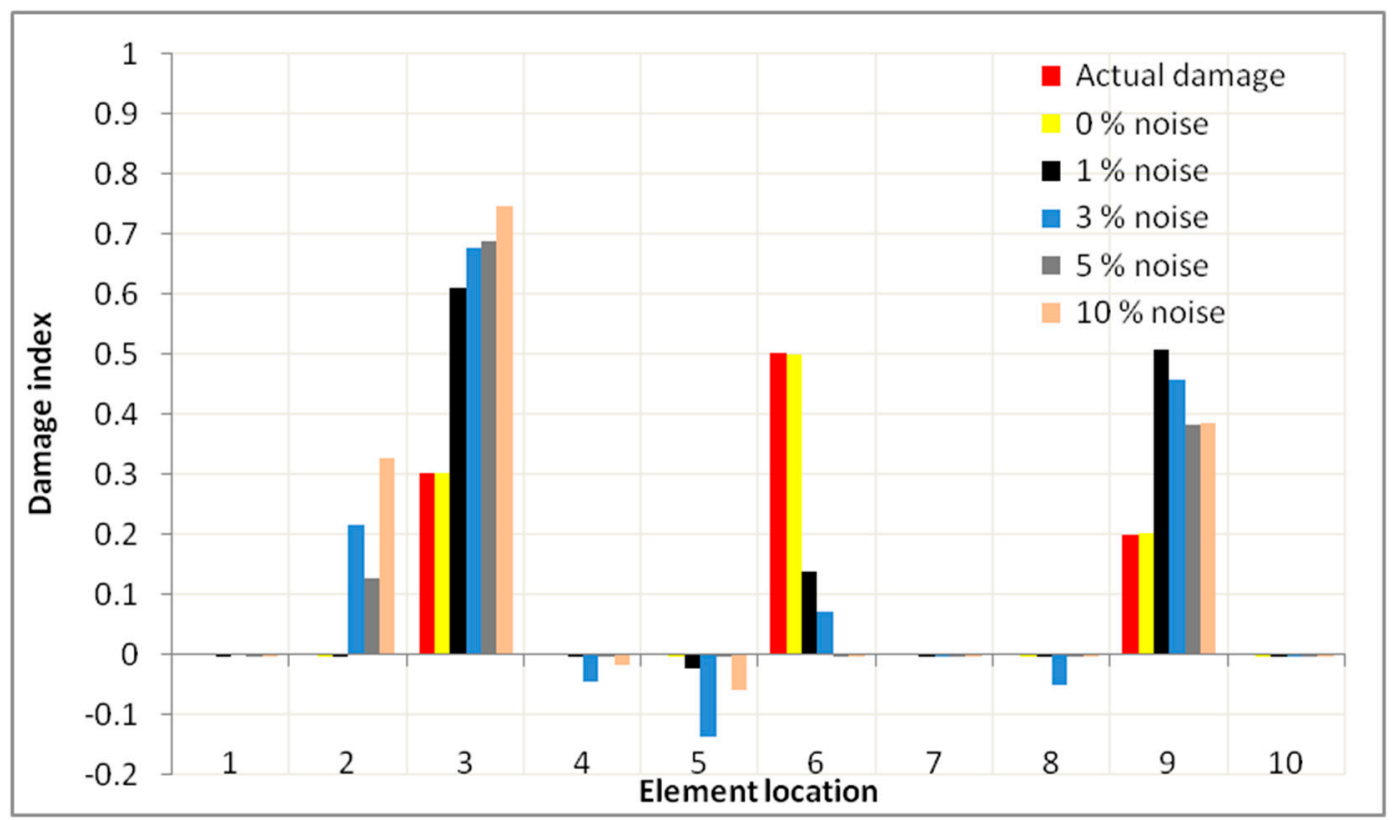

(b)

Figure 14. Damage distribution for Case 2 using Objective Function III with regularization for (a) noise both in frequency and mode shapes and (b) noise in mode shapes only.

Later on, model updating was again done with all three objective functions with regularization, and the results are given in Figure 15b. The damage index for Element No. 9 was found to be 0.61, 0.58 and 0.43 (actual damage index $=0.875$ ) for regularized Objective Functions I, II and III, respectively. Likewise, wrong detections in other elements were less in the regularized Objective Function I than the 
other two. The results show that the method proposed in the paper is efficient and utilizes the benefits of both EA, as well as the regularization function by giving much better damage assessment. It can be seen that better results are obtained with the proposed approach as compared to other researchers using this example $[16,45]$.

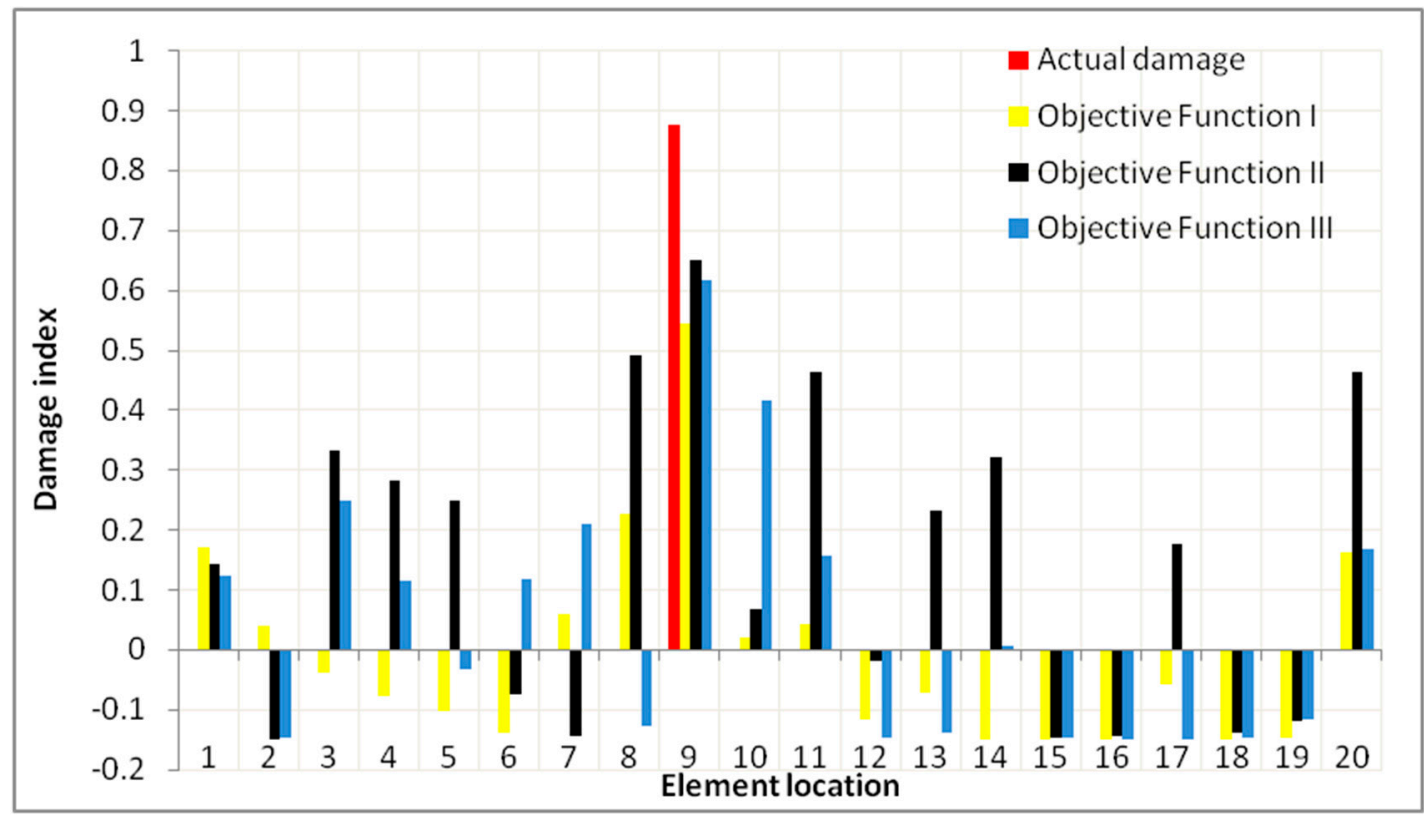

(a)



(b)

Figure 15. Damage distribution for Case 3 using (a) Objective Functions I, II and III without regularization and (b) Objective Functions I, II and III with regularization.

\section{Conclusions}

In this research, three different objective functions based on frequencies, MAC, modal strain energy and flexibility have been analyzed for damage detection. Two simulated beams and an 
experimental beam were investigated to check the performance of these objective functions using GA. The following conclusions were drawn from the study:

1. The simulated beam (Case 1) was investigated with a single damage scenario. First, three mode shapes have been considered for damage assessment for the different objective functions. Two different noise cases, i.e., noise in both frequencies and mode shapes and noise in mode shapes only at $1 \%, 3 \%, 5 \%$ and $10 \%$ have been investigated in the frequency domain. It has been found that with no noise, all the objective functions performed well and detected the damage correctly. However, results indicate that with an increase in the noise, the damage detection capabilities of all the objective functions decreased. Objective Function I based on frequencies and MAC has worked better in damage detection than Objective Functions II and III in noisy conditions. However, less degradation has been seen in the noise in mode shapes-only case as compared to noise both in frequencies and mode shapes. Probable reasons for the better performance of Objective Function I as compared to the other two were also discussed. It was found that the function value of Objective Function I is lesser than Objective Functions II and III.

2. A regularization function was added in the objective functions based on the a priori modeling of the structure. The multi-objective GA was used to find the optimal tradeoff between the objective function and the regularization part. The results show that the regularization function performed well even in noisy conditions for Objective Function I.

3. The multiple damage scenario has been simulated in Case 2 where three elements were damaged. At $0 \%$ noise, all the functions detected the damage correctly. However, the performance degraded when noise levels were increased. The regularization function has performed well for Objective Function I only, which proves its adequacy in multiple damage scenarios.

4. The simulations were later verified on an experimentally tested beam. Convergence analysis of the SAP 2000 model was performed for the first three natural frequencies, and the 20-node beam was selected for the FE model. It was found that detections in non-damaged elements were of greater magnitude for Objective Functions II and III as compared to Objective Function I. Regularization was attempted for the experimental beam. The damage index was much improved for the damaged, as well as for the undamaged elements for Objective Function I with regularization, which further proves the performance of the proposed approach in actual experimental conditions.

The paper has proposed the use of EA for beam structures. Further studies on 3D complex structures where noise has been added directly to time series data are recommended.

Author Contributions: Faisal Shabbir conceived of the idea of the paper and designed the experiments. Muhammad Imran Khan contributed towards simulation tools. Naveed Ahmed helped in the simulations. Faisal Shabbir and Muhammad Fiaz Tahir analyzed the data. Naeem Ejaz and Jawad Hussain helped in the revisions of the paper.

Conflicts of Interest: The authors declare no conflict of interest.

\section{References}

1. Brownjohn, J.M.W.; Xia, P.Q.; Hao, H.; Xia, Y. Civil structure condition assessment by FE model updating: Methodology and case studies. Finite Elem. Anal. Des. 2001, 37, 761-775. [CrossRef]

2. Moon, F.L.; Aktan, A.E. Impacts of epistemic (bias) uncertainty on structural identification of constructed (civil) systems. Shock Vib. Dig. 2006, 38, 399-420. [CrossRef]

3. Aktan, A.E.; Helmicki, A.J.; Hunt, V.J. Issues in health monitoring for intelligent infrastructure. Smart Mater. Struct. 1998, 7, 674-692. [CrossRef]

4. Aktan, A.E.; Catbas, F.N.; Grimmelsman, K.A.; Tsikos, C.J. Issues in infrastructure health monitoring for management. J. Eng. Mech. ASCE 2000, 126, 711-724. [CrossRef]

5. Ward, H.; Heylen, W. Modal Analysis Theory and Testing; Lammens, S., Sas, P., Eds.; Katholieke Universiteit Leuven: Leuven, Belgium, 1997. 
6. Wu, Y.-X. (Ed.) Sensitivity-Based Finite Element Model Updating Methods with Applications to Electronic Equipments. Ph.D. Thesis, Faculte Polytechnique de Mons, Mons, Belgium, 1999.

7. Kong, X.; Cai, C.-S.; Hu, J. The State-of-the-art on framework of vibration-based structural damage identification for decision making. Appl. Sci. 2017, 7, 497. [CrossRef]

8. Horst, R.; Pardalos, P.M.; Thoai, N.V. Introduction to Global Optimization; Springer: Dordrecht, The Netherlands, 2000.

9. Yu, E.; Taciroglu, E.; Wallace, J.W. Parameter identification of framed structures using an improved finite element model-updating method-Part I: Formulation and verification. Earthq. Eng. Struct. Dyn. 2007, 36, 619-639. [CrossRef]

10. Zivanovic, S.; Pavic, A.; Reynolds, P. Finite element modelling and updating of a lively footbridge: The complete process. J. Sound Vib. 2007, 301, 126-145. [CrossRef]

11. Jaishi, B.; Ren, W.X. Finite element model updating based on eigenvalue and strain energy residuals using multiobjective optimisation technique. Mech. Syst. Signal Process. 2007, 21, 2295-2317. [CrossRef]

12. Jaishi, B.; Ren, W.-X. Damage detection by finite element model updating using modal flexibility residual. J. Sound Vib. 2006, 290, 369-387. [CrossRef]

13. Kalyanmoy, D.; Deb, K. Multi-Objective Optimization Using Evolutionary Algorithms; John Wiley \& Sons: Chichester, NY, USA, 2001.

14. Tebaldi, A.; Dos Santos Coelho, L.; Lopes, V., Jr. Detection of damage in intelligent structures using optimization by a particle swarm: Fundamentals and case studies. Controle Autom. Soc. Bras. Autom. 2006, 17, 312-330. [CrossRef]

15. Tu, Z.; Lu, Y. FE model updating using artificial boundary conditions with genetic algorithms. Comput. Struct. 2008, 86, 714-727. [CrossRef]

16. Perera, R.; Torres, R. Structural damage detection via modal data with genetic algorithms. J. Struct. Eng. ASCE 2006, 132, 1491-1501. [CrossRef]

17. Raich, A.M.; Liszkai, T.R. Improving the performance of structural damage detection methods using advanced genetic algorithms. J. Struct. Eng. ASCE 2007, 133, 449-461. [CrossRef]

18. Perera, R.; Ruiz, A.; Manzano, C. Performance assessment of multicriteria damage identification genetic algorithms. Comput. Struct. 2009, 87, 120-127. [CrossRef]

19. Levin, R.I.; Lieven, N.A.J. Dynamic finite element model updating using simulated annealing and genetic algorithms. Mech. Syst. Signal Process. 1998, 12, 91-120. [CrossRef]

20. Saada, M.M.; Arafa, M.H.; Nassef, A.O. Finite element model updating approach to damage identification in beams using particle swarm optimization. In Proceedings of the 34th Design Automation Conference, Brooklyn, NY, USA, 3-6 August 2008.

21. Perera, R.; Ruiz, A. A multistage FE updating procedure for damage identification in large-scale structures based on multiobjective evolutionary optimization. Mech. Syst. Signal Process. 2008, 22, 970-991. [CrossRef]

22. Hoseini Vaez, S.R.; Fallah, N. Damage detection of thin plates using GA-PSO algorithm based on modal data. Arabian J. Sci. Eng. 2017, 42, 1251-1263. [CrossRef]

23. Fabbrocino, F.; Farina, I.; Berardi, V.P.; Ferreira, A.J.M.; Fraternali, F. On the thrust surface of unreinforced and FRP-/FRCM-reinforced masonry domes. Compos. Part B Eng. 2015, 83, 297-305. [CrossRef]

24. He, R.-S.; Hwang, S.-F. Damage detection by an adaptive real-parameter simulated annealing genetic algorithm. Comput. Struct. 2006, 84, 2231-2243. [CrossRef]

25. Jin, S.-S.; Cho, S.; Jung, H.-J.; Lee, J.-J.; Yun, C.-B. A new multi-objective approach to finite element model updating. J. Sound Vib. 2014, 333, 2323-2338. [CrossRef]

26. Begambre, O.; Laier, J.E. A hybrid particle swarm optimization-Simplex algorithm (PSOS) for structural damage identification. Adv. Eng. Softw. 2009, 40, 883-891. [CrossRef]

27. Perera, R.; Fang, S.E.; Ruiz, A. Application of particle swarm optimization and genetic algorithms to multiobjective damage identification inverse problems with modelling errors. Meccanica 2010, 45, 723-734. [CrossRef]

28. Yeo, I.; Shin, S.; Lee, H.S.; Chang, S.-P. Statistical damage assessment of framed structures from static responses. J. Eng. Mech. 2000, 126, 414-421. [CrossRef]

29. Zhang, Q.; Chang, C.; Chang, T. Finite element model updating for structures with parametric constraints. Earthq. Eng. Struct. Dyn. 2000, 29, 927-944. [CrossRef] 
30. Zang, C.; Chen, G.; Ewins, D. Finite element model updating with modal data. In Proceedings of the 24th International Modal Analysis Conference, Saint Louis, MO, USA, 30 January-2 February 2006.

31. Li, S.; Brown, D.L. Application of a unified matrix polynomial approach (UMPA) to perturbed boundary condition (PBC) testing. Mech. Syst. Signal Process. 1995, 9, 77-84. [CrossRef]

32. Schnur, D.; Zabaras, N. Finite element solution of two-dimensional inverse elastic problems using spatial smoothing. Int. J. Numer. Methods Eng. 1990, 30, 57-75. [CrossRef]

33. Tikhonov, A.N.; Goncharsky, A.; Stepanov, V.; Yagola, A.G. Numerical Methods for the Solution of Ill-Posed Problems; Springer: Berlin, Germany, 1995; Volume 328, pp. 7-63.

34. Ahmadian, H.; Mottershead, J.E.; Friswell, M.I. Regularisation methods for finite element model updating. Mech. Syst. Signal Process. 1998, 12, 47-64. [CrossRef]

35. Weber, B.; Paultre, P.; Proulx, J. Structural damage detection using nonlinear parameter identification with Tikhonov regularization. Struct. Control Health Monit. 2007, 14, 406-427. [CrossRef]

36. Weber, B.; Paultre, P.; Proulx, J. Consistent regularization of nonlinear model updating for damage identification. Mech. Syst. Signal Process. 2009, 23, 1965-1985. [CrossRef]

37. Titurus, B.; Friswell, M.I. Regularization in model updating. Int. J. Numer. Methods Eng. 2008, 75, 440-478. [CrossRef]

38. Mottershead, J.E.; Link, M.; Friswell, M.I. The sensitivity method in finite element model updating: A tutorial. Mech. Syst. Signal Process. 2011, 25, 2275-2296. [CrossRef]

39. Chen, H.-P.; Maung, T.S. Regularised finite element model updating using measured incomplete modal data. J. Sound Vib. 2014, 333, 5566-5582. [CrossRef]

40. Entezami, A.; Shariatmadar, H.; Sarmadi, H. Structural damage detection by a new iterative regularization method and an improved sensitivity function. J. Sound Vib. 2017, 399, 285-307. [CrossRef]

41. Grip, N.; Sabourova, N.; Tu, Y. Sensitivity-based model updating for structural damage identification using total variation regularization. Mech. Syst. Signal Process. 2017, 84, 365-383. [CrossRef]

42. Friswell, M.I.; Mottershead, J.E. Finite Element Model Updating in Structural Dynamics; Kluwer Academic Publishers: Dordrecht, The Netherlands, 1995.

43. Möller, P.W.; Friberg, O. Updating large finite element models in structural dynamics. AIAA J. 1998, 36, 1861-1868. [CrossRef]

44. Lemaitre, J. A Course on Damage Mechanics; Springer: Berlin, Germany, 2012.

45. Hu, N.; Wang, X.; Fukunaga, H.; Yao, Z.H.; Zhang, H.X.; Wu, Z.S. Damage assessment of structures using modal test data. Int. J. Solids Struct. 2001, 38, 3111-3126. [CrossRef]

46. Friswell, M.I.; Penny, J.E.T. Crack modeling for structural health monitoring. Struct. Health Monit. 2002, 1, 139-148. [CrossRef]

47. Sinha, J.K.; Friswell, M.I.; Edwards, S. Simplified models for the location of cracks in beam structures using measured vibration data. J. Sound Vib. 2002, 251, 13-38. [CrossRef]

48. Shabbir, F.; Omenzetter, P. Model updating using genetic algorithms with sequential niche technique. Eng. Struct. 2016, 120, 166-182. [CrossRef]

49. Pastor, M.; Binda, M.; Harčarik, T. Modal assurance criterion. Procedia Eng. 2012, 48, 543-548. [CrossRef]

50. Farrar, C.R.; Jauregui, D.A. Comparative study of damage identification algorithms applied to a bridge: II. Numerical study. Smart Mater. Struct. 1998, 7, 720. [CrossRef]

(C) 2017 by the authors. Licensee MDPI, Basel, Switzerland. This article is an open access article distributed under the terms and conditions of the Creative Commons Attribution (CC BY) license (http://creativecommons.org/licenses/by/4.0/). 